Article

\title{
Multi-Scroll Chaotic Attractors in SC-CNN via Hyperbolic Tangent Function
}

\author{
Enis Günay ${ }^{1, *}$ and Kenan Altun ${ }^{2}$ \\ 1 Department of Electrical and Electronics Engineering, Erciyes University, 38039 Kayseri, Turkey \\ 2 Sivas Vocational College, Cumhuriyet University, 58140 Sivas, Turkey; kaltun@cumhuriyet.edu.tr \\ * Correspondence: egunay@erciyes.edu.tr; Tel.:+90-352-207-6666 (ext. 32232)
}

Received: 9 April 2018; Accepted: 7 May 2018; Published: 9 May 2018

check for updates

\begin{abstract}
A State Controlled-Cellular Neural Network (SC-CNN) based chaotic model for generating multi-scroll attractors via hyperbolic tangent function series is proposed in this paper. After presenting the double scroll generation, the presented SC-CNN system is used in multi-scroll chaotic attractor generation by adding hyperbolic tangent function series. By using equilibrium analysis and their stability such as Lyapunov exponent analysis, bifurcation diagrams and Poincaré map, the dynamical behaviors of the proposed system are theoretically analyzed and numerically investigated.
\end{abstract}

Keywords: State Controlled-Cellular Neural Networks; multi-scroll chaotic attractors; hyperbolic tangent function

\section{Introduction}

It has been accepted that chaos can be quite useful in some engineering and technological applications. Complex biological systems, information processing, secure communication, mechanism of memory, etc., can be listed among these applications. Chaos, when under control, can provide useful properties and flexibility to the designer. Some studies have been carried out to elucidate the effects of memory on the excitation dynamics of organic dynamical systems. For example, cardiac action potential models demonstrate that memory can cause dynamical instabilities which result in complex excitation dynamics and chaos [1]. Moreover, chaos-based communication schemes and chaos-based cryptosystems using more complex attractors are preferable for enhancing the security of the systems. Accordingly, simple dynamical systems like multi-scroll chaos generators that show more complex chaotic attractors are desirable [2].

Cellular Neural Network (CNN), introduced by Chua and Yang in 1988, has been the subject of many theoretical and experimental studies as a subfield of nonlinear electrical systems [3,4]. Various $\mathrm{CNN}$ based chaotic oscillators have facilitated the understanding of the chaotic phenomenon by complementing the research on chaos through analog simulations. Designing more complex chaotic attractors using CNN among multi-scroll generation studies has yet to be well studied and it needs proper consideration.

The work on generating multi-scroll attractors has always grab researchers' attention and has gradually become a new research field. The functions such as piecewise linear (PWL), sawtooth, step wave, hysteresis series, switching, sine, saturated sequence and hyperbolic tangent have been proposed for generating a different type of multi-scroll chaotic attractors so far.

Suykens and Vandewalle proposed quasi-linear function approach to introduce a family of n-double scroll chaotic attractors [5-7]. Alaoui et al. presented multispiral chaotic attractors using PWL function approach for both autonomous and non-autonomous differential equations [8,9]. Yalcin et al. introduced a hyperchaotic attractor technique for generating a family of multi-scroll, and they also proposed a simple circuit model for generating multi-scroll chaotic attractors [10,11]. Lü et al. 
proposed multi-scroll chaotic attractors using hysteresis series method, saturated function series approach and thresholding approach [12-14]. Sine function method by Tang et al. and nonlinear trans conductor method by Özoğuz et al. and Salama et al. are suggested for creating n-scroll chaotic attractors [15-17]. For creating grid scroll hyperchaotic attractors from one-directional (1-D) n-scroll to three-directional (3-D) n-scroll attractors, Cafagna and Grassi developed a coupling Chua's circuit method $[18,19]$. Yalcin et al. presented a family of scroll-grid chaotic attractors using a stepping circuit [20]. Adjustable triangular, sawtooth and trans conductor wave functions were also utilized by Yuet al. to generate n-scroll chaotic attractors from a general jerk circuit [21,22]. Deng and Lü used a fractional order system such as stair function, saturated, and hysteresis series methods to generate n-scroll attractors [23-25]. Generation of multi-scroll chaotic attractors for the fractional-order system using the piecewise-linear, the stair, and the saturated function are utilized by Chen et al. [26,27]. While $\mathrm{Xu}$ and $\mathrm{Yu}$ presented hyperbolic tangent function in chaos control and chaos synchronization of multi-scroll chaotic attractors, Chen et al. used hyperbolic tangent function series to present grid multi-scroll chaos generation [28,29]. Wang et al. showed multi-level pulses, multi-double-scroll attractors that are generated from the variable-boostable chaotic system [30]. Muñoz-Pacheco et al. presented experimental verification of optimized multi-scroll chaotic attractors based on irregular saturated function [31].

Itis shown that multi-scrolls could be generated by adding additional breaking points in the output function of SC-CNN, which has a PWL characteristic [32]. Günay and Alçı revisited multi-scrolls in SC-CNN circuit via diode-based PWL function [33]. In addition to PWL approach, the trigonometric function was presented by Günay and Kılıç as an alternative way of generating multi-scroll attractors in SC-CNN [34].

In this paper, a novel methodfor multi-scroll chaotic attractor generation in SC-CNN is presented based on hyperbolic tangent function. Firstly, the double scroll generation is analyzed via dynamical behaviors of the presented system, such as its equilibrium, stability, Lyapunov exponents, bifurcation diagrams, and Poincaré map. Then, the dynamical mechanisms of multi-scroll chaos generations based on the corresponding model are theoretically analyzed and numerically investigated including one, two, and three directional multi-scroll chaotic attractors. Finally, discussions, suggestions, and potential future work plans are presented in the Conclusions Section.

\section{New Double Scroll Attractor}

As stated in the literature, besides being an image processing system, Cellular Neural Networks (CNNs) also offer an effective methodology and technology for the analysis and design of complex dynamics [2]. In 1995, Arena et al. showed complex dynamical systems can be imitated by CNN canonic model with an additional input that represents the feedbacks from the states of the cells [34]. The generalized dimensionless nonlinear state equations of SC-CNN can be given as follows:

$$
\dot{x}_{j}=-x_{j}+a_{j} y_{j}+G_{\mathrm{o}}+G_{s}+i_{j}, y_{j}=\frac{1}{2}\left[\left|x_{j}+1\right|-\left|x_{j}-1\right|\right]
$$

where $j, x_{j}$, and $y_{j}$ denote cell index, state variable and cell output, respectively. $a_{j}$ and $i_{j}$ stand for constant parameter and threshold, respectively. $G_{O}$ and $G_{S}$ represent the outputs and state variables of connected cells, respectively. The dynamic model of three fully connected generalized CNN cells according to Equation (1) is defined in [34] as follows:

$$
\begin{aligned}
& \dot{x}_{1}=-x_{1}+\sum_{k=1}^{3} a_{1 k} y_{k}+\sum_{k=1}^{3} s_{1 k} x_{k}+i_{1} \\
& \dot{x}_{2}=-x_{2}+\sum_{k=1}^{3} a_{2 k} y_{k}+\sum_{k=1}^{3} s_{2 k} x_{k}+i_{2} \\
& \dot{x}_{3}=-x_{3}+\sum_{k=1}^{3} a_{3 k} y_{k}+\sum_{k=1}^{3} s_{3 k} x_{k}+i_{3}
\end{aligned}
$$


where $k$ is the cell index.

In this section, we discuss new double scroll generation and give its dynamical properties such as their equilibria and stability. Consider the dynamical system obtained from Equation (2):

$$
\begin{gathered}
\dot{x}_{1}=x_{2}+x_{3} \\
\dot{x}_{2}=-x_{1}-x_{2}+s_{22} x_{2} \\
\dot{x}_{3}=-x_{3}-\tanh \left(n y_{2}\right)+i_{3} \\
y_{2}=\frac{1}{2}\left[\left|x_{2}+1\right|-\left|x_{2}-1\right|\right]
\end{gathered}
$$

where

$$
\begin{gathered}
s_{11}=s_{12}=s_{13}=a_{32}=1 ; s_{21}=-1 ; \\
s_{23}=s_{31}=s_{32}=s_{33}=a_{11}=a_{12}=a_{13}=a_{21}=a_{22}=a_{23}=a_{31}=a_{33}=i_{1}=i_{2}=0 ; \text { and }
\end{gathered}
$$

$s_{22}, i_{3}$ and $n$ are constants. The equilibrium points of Equation (3) exist in three subspaces defined as follows:

$$
\begin{gathered}
D_{+}=\left\{\left(x_{1}, x_{2}, x_{3}\right) \mid x_{2} \geq 1\right\}: P_{+}=\left(k_{\alpha}, k_{\beta}, k_{\gamma}\right), \\
D_{*}=\left\{\left(x_{1}, x_{2}, x_{3}\right)|| x_{2} \mid \leq 1\right\}: P_{*}=\left(l_{\alpha}, l_{\beta}, l_{\gamma}\right) \\
D_{-}=\left\{\left(x_{1}, x_{2}, x_{3}\right) \mid x_{2} \leq-1\right\}: P_{-}=\left(m_{\alpha}, m_{\beta}, m_{\gamma}\right)
\end{gathered}
$$

where

$$
\begin{gathered}
k_{\alpha}=-\left(s_{22}-1\right)\left(i_{3}-1\right), k_{\beta}=1-i_{3}, k_{\gamma}=i_{3}-1 \\
m_{\alpha}=-\left(s_{22}-1\right)\left(i_{3}+1\right), m_{\beta}=-1-i_{3}, m_{\gamma}=i_{3}+1 \\
l_{\alpha}=\frac{i_{3}\left(s_{22}-1\right)}{(n+1)}, l_{\beta}=\frac{i_{3}}{(n+1)}, l_{\gamma}=\frac{-i_{3}}{(n+1)}
\end{gathered}
$$

The equilibrium points $P_{+}, P_{*}$, and $P_{-}$can be found from the Jacobian matrices.

$$
J_{+}=J_{-}=\left[\begin{array}{ccc}
0 & 1 & 1 \\
-1 & \left(s_{22}-1\right) & 0 \\
0 & 0 & -1
\end{array}\right] ; J_{*}=\left[\begin{array}{ccc}
0 & 1 & 1 \\
-1 & \left(s_{22}-1\right) & 0 \\
0 & -n & -1
\end{array}\right]
$$

The corresponding characteristic equation is:

$$
a \lambda^{3}+b \lambda^{2}+c \lambda+d=0
$$

where

$$
\begin{gathered}
p=\frac{c}{a}-\frac{b^{2}}{3 a^{2}}, q=\frac{d}{a}-\frac{b c}{3 a^{2}}+\frac{2 b^{3}}{27 a^{3}} \\
\lambda_{1}=\frac{-b}{3}+\sqrt[3]{\frac{-q^{2}}{2}}+\sqrt{\Delta}+\sqrt[3]{\frac{-q^{2}}{2}-\sqrt{\Delta}} \\
\lambda_{2,3}=\frac{-b}{3}-\frac{1}{2}\left(\sqrt[3]{\frac{-q^{2}}{2}+\sqrt{\Delta}}+\sqrt[3]{\frac{-q^{2}}{2}-\sqrt{\Delta}}\right) \pm \frac{i \sqrt{3}}{2}\left(\sqrt[3]{\frac{-q^{2}}{2}+\sqrt{\Delta}}+\sqrt[3]{\frac{-q^{2}}{2}-\sqrt{\Delta}}\right) \equiv \alpha \pm \beta i, \\
\Delta=\frac{d b^{3}}{27}-\frac{b^{2} c^{2}}{108}-\frac{b c d}{6}+\frac{c^{3}}{27}+\frac{d^{2}}{4}
\end{gathered}
$$

The equilibrium points $P_{+}, P_{*}$, and $P_{-}$are given by:

$$
\begin{gathered}
P_{ \pm}(\lambda)=\lambda^{3}+\left(1-s_{22}\right) \lambda^{2}+\left(1-s_{22}\right) \lambda+1 \\
P_{*}(\lambda)=\lambda^{3}+\left(1-s_{22}\right) \lambda^{2}+\left(1-s_{22}\right) \lambda+n+1
\end{gathered}
$$

Numerical computations show that proposed system in Equation (3) will produce chaotic behavior under the conditions of $\lambda_{1}<0,\left(1<s_{22}<1.28\right),\left(-1<i_{3}<1\right)$ and $n>0$. Thus, Equation (3) has a negative eigenvalue and one pair of complex conjugate eigenvalues with positive real parts. Thus, the proposed 
SC-CNN system is unstable, and all equilibrium points $P$ are saddle points of index 2 [35]. Those can be given as follows:

$$
\begin{gathered}
\Delta=\frac{d b^{3}}{27}-\frac{b^{2} c^{2}}{108}-\frac{b c d}{6}+\frac{c^{3}}{27}+\frac{d^{2}}{4}>0 ; \lambda_{1}=\frac{-b}{3}+\sqrt[3]{\frac{-q^{2}}{2}+\sqrt{\Delta}}+\sqrt[3]{\frac{-q^{2}}{2}-\sqrt{\Delta}}<0 \\
\alpha=\frac{-b}{3}-\frac{1}{2}\left(\sqrt[3]{\frac{-q^{2}}{2}+\sqrt{\Delta}}+\sqrt[3]{\frac{-q^{2}}{2}-\sqrt{\Delta}}\right)>0
\end{gathered}
$$

The system can produce chaotic behaviors for most of the initial conditions and those are taken as $(0.1,0.1,0.1)$ in this paper. The equilibrium points and eigenvalues of the Jacobian matrices are calculated for the parameter values, namely: $s_{11}=s_{12}=s_{13}=a_{32}=1 ; s_{21}=-1 ; s_{22}=1.2 ; i_{33}=0.1 ;$ and $n$ $=10$. The eigenvalues for equilibrium point $(-0.06,-0.05,0.05) \in D^{*}$ are calculated from the matrix $J^{*}$ as $\lambda_{1}=-1.2469$ and $\lambda_{2,3}=0.7234 \pm 1.0396 i$.

On the other hand, the eigenvalues for equilibrium points $(-1.32,-1.1,1.1) \in D_{+}$and $(1.08,0.9$, $-0.9) \in D_{-}$are calculated from the matrix $J_{ \pm}$as $\lambda_{1}=-1$ and $\lambda_{2,3}=0.6 \pm 0.8 i$. As seen from results, the proposed system has one negative root and one pair of complex conjugate roots with positive real parts. Thus, SC-CNN system is unstable, and all equilibrium $P$ are saddle points of index 2 .

To study the dynamics of the system in Equation (3), firstly, phase portraits and time domain responses are presented in the following figures. In phase portraits, 3T-periodic solution for $i_{33}=-0.83$, one band chaotic solution for $i_{33}=-0.59$, a double-scroll chaotic attractor for $i_{33}=0.1$, and similarly 3T-periodic solution for $i_{33}=0.83$ can be seen in Figure 1a-h with time domain representations.

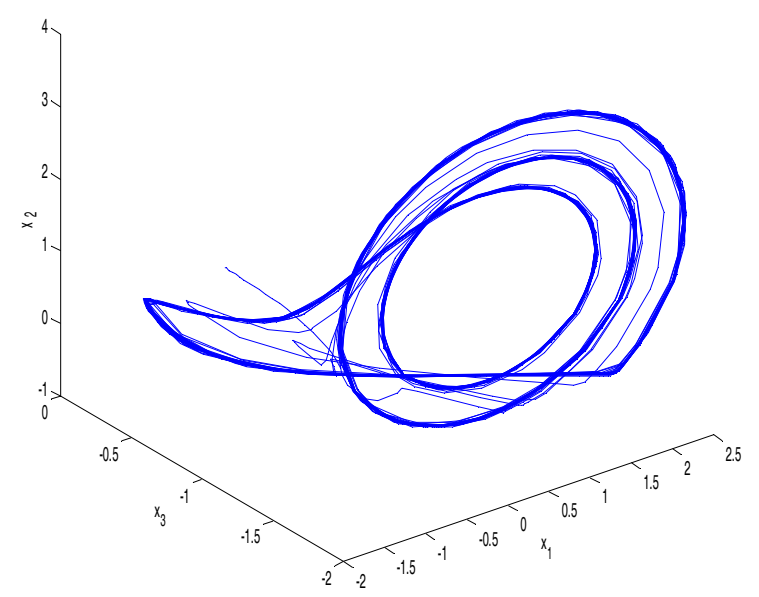

(a)

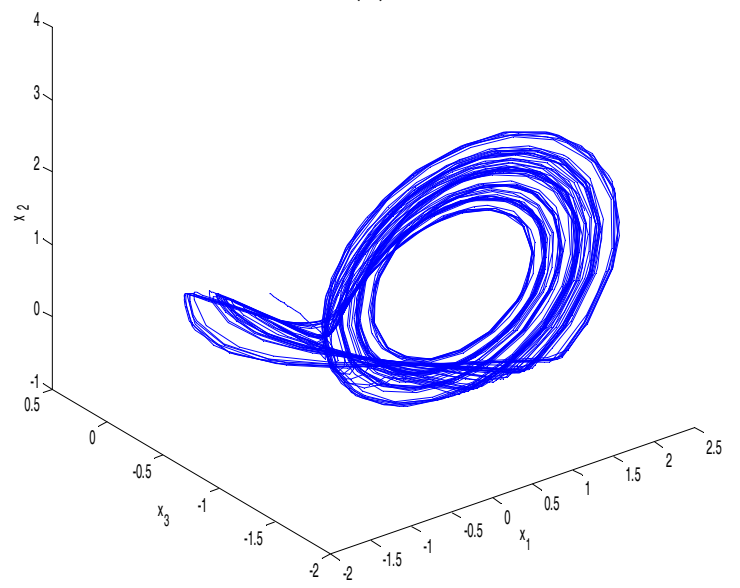

(c)

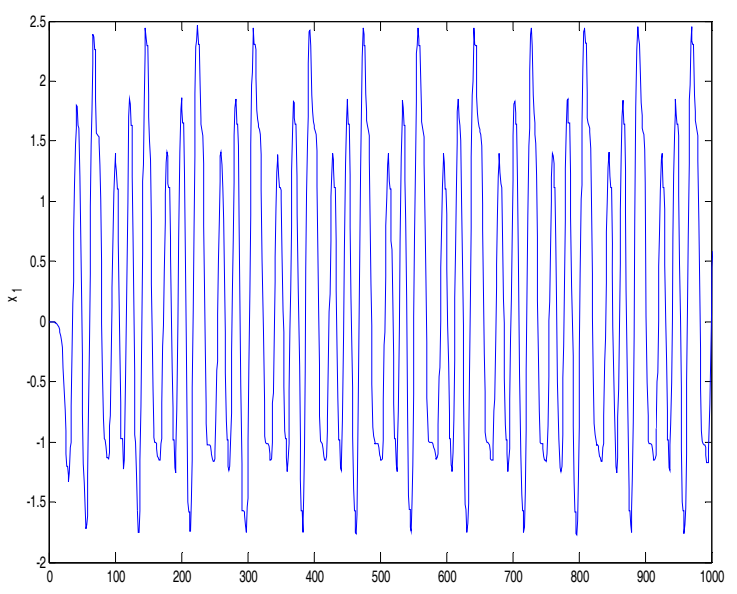

(b)

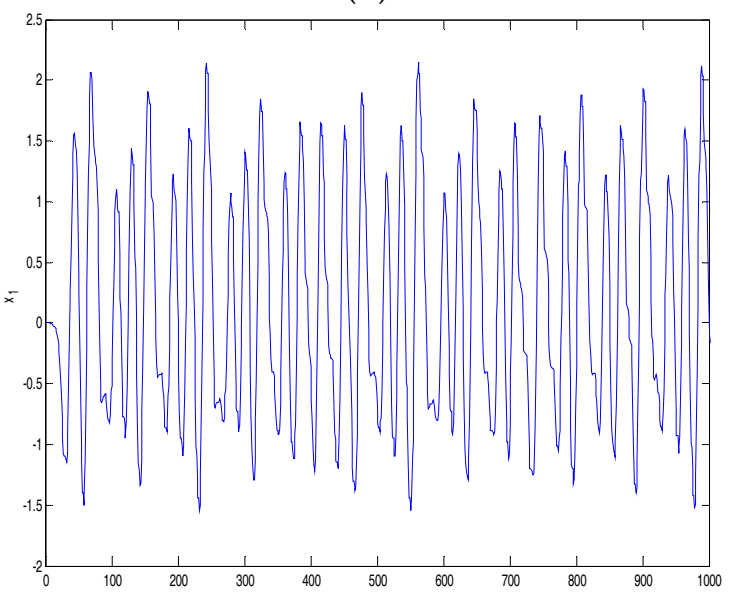

(d)

Figure 1. Cont. 


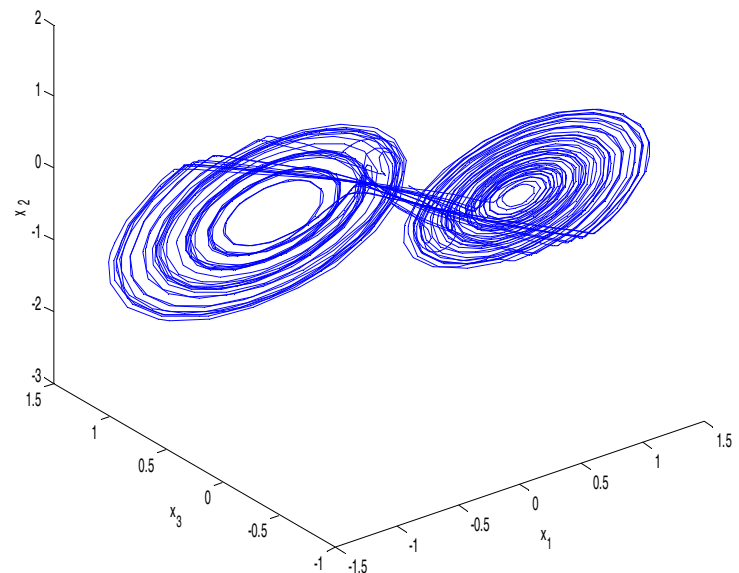

(e)

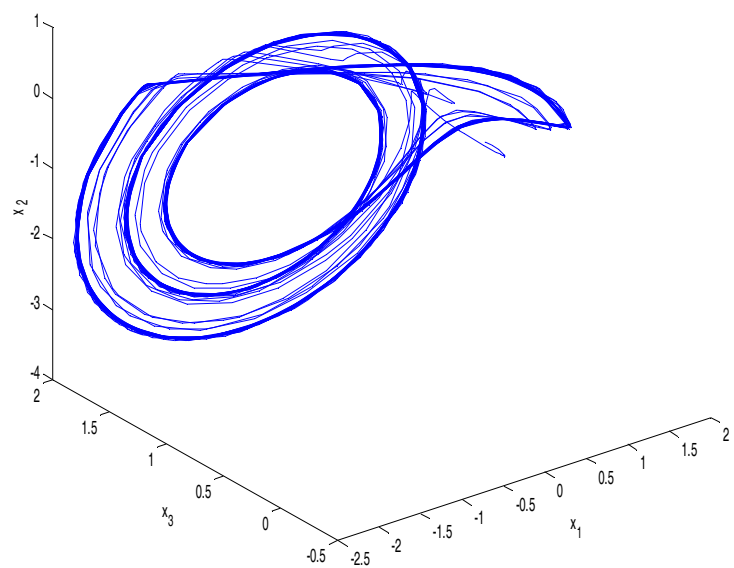

(g)

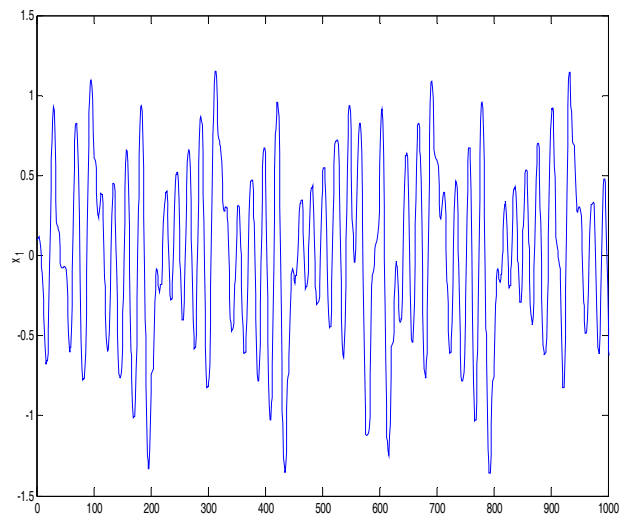

(f)

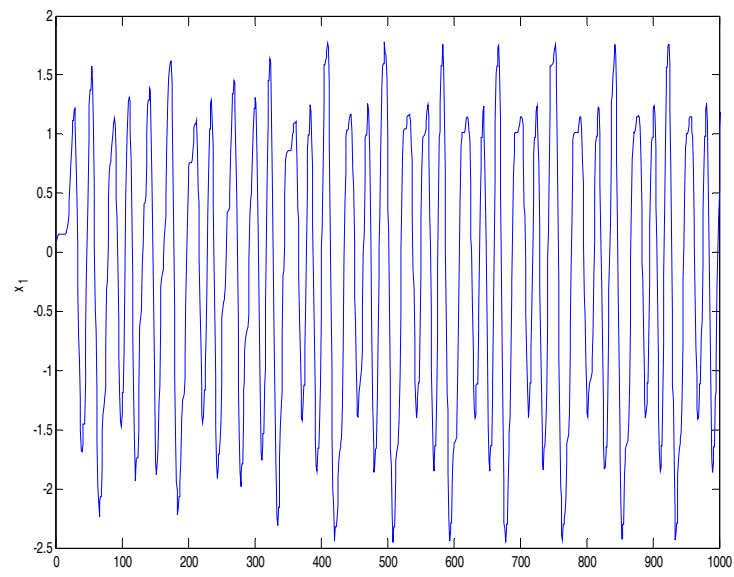

(h)

Figure 1. Numerical results of the Switched-SC-CNN Based system: (a) 3T-periodic solution for $i_{33}$ $=-0.83 ;(\mathbf{b})$ time response of $x_{1}(\mathrm{t})$ dynamic for $i_{33}=-0.83$; (c) one band chaos for $i_{33}=-0.59 ;(\mathbf{d})$ time response of $x_{1}(\mathrm{t})$ dynamic for $i_{33}=-0.59 ;(\mathbf{e})$ double scroll for $i_{33}=0.1 ;(\mathbf{f})$ time response of $x_{2}(\mathrm{t})$ dynamic for $i_{33}=0.1 ;(\mathbf{g}) 3 \mathrm{~T}$-periodic solution for $i_{33}=0.83$; and (h) time response of $x_{1}(\mathrm{t})$ dynamic for $i_{33}=0.83$.

\section{Bifurcation Diagrams, Lyapunov Exponents Spectra and Poincaré Map}

In this study, three bifurcation studies are given. In the first one, system parameters are fixed as $s_{11}=s_{12}=s_{13}=a_{32}=1 ; s_{21}=-1 ; s_{22}=1.2 ;$ and $n=10$, with varying $i_{3}$. The system is calculated numerically for $i_{3} \in[-1,1]$, and an increment of $\Delta i_{3}=0.001$. Period-doubling route to chaos is clearly seen in the bifurcation diagram, $x_{1}$ versus $i_{3}$, as given in Figure 2a. From the bifurcation diagram and the phase domain figures given in Figure 1, the proposed system is symmetrical with respect to $i_{3}$.

In the second bifurcation study, system parameters are fixed as $s_{11}=s_{12}=s_{13}=a_{32}=1 ; s_{21}=-1$; $i_{3}=0.1$; and $n=10$, with varying $s_{22}$. The system is calculated numerically with $s_{22} \in[1,1.28]$ for an increment of $\Delta s_{22}=0.001$. Within [1, 1.28], various curves seem to expand explosively and merge together to produce an area of almost solid black, which are indicators of the onset of chaos.

In the last bifurcation study, system parameters are fixed as $s_{11}=s_{12}=s_{13}=a_{32}=1 ; s_{21}=-1$; $i_{3}=0.1$; and $s_{22}=1.2$, with varying $n$. The system is calculated numerically with $n \in[1,3.5]$ for an increment of $\Delta n=0.001$. As shown in Figure 2c, when $n$ exceeds 1.21, period-1 orbit becomes, when $n$ exceeds 1.85 period-2 orbit, becomes period-4 orbit. 


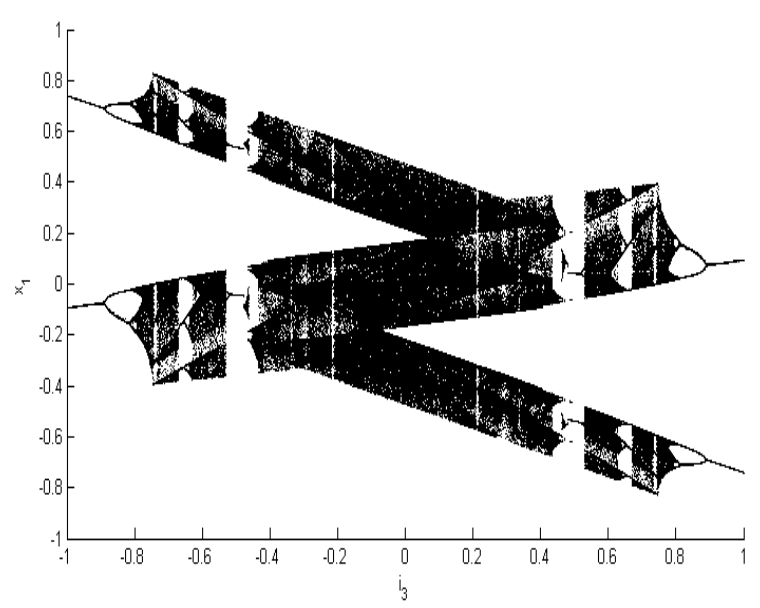

(a)

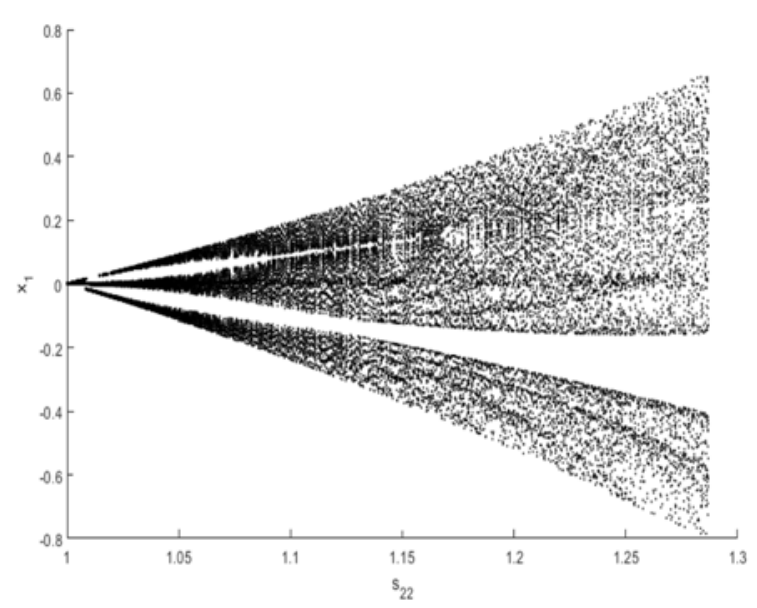

(b)

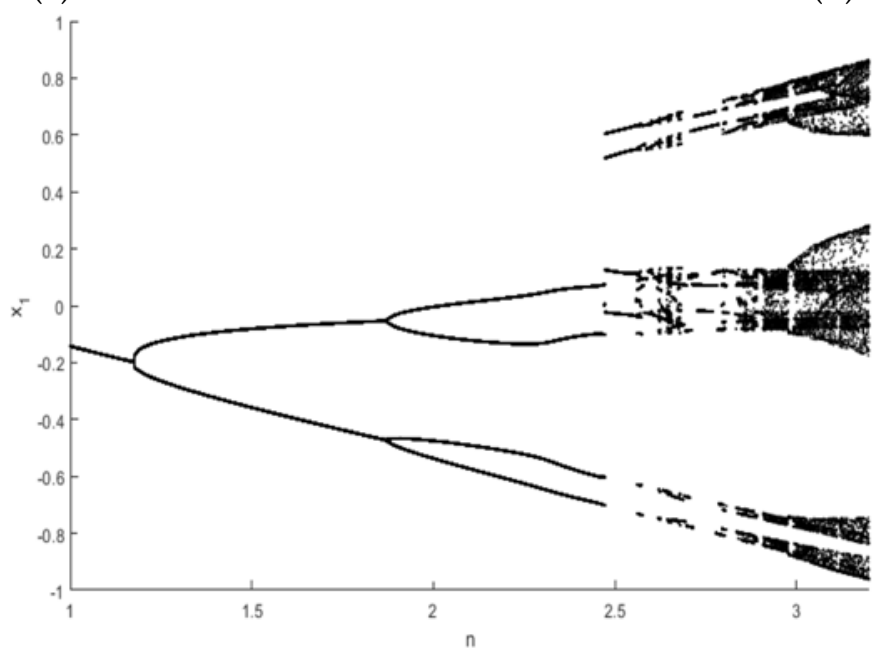

(c)

Figure 2. (a) Bifurcation study with $x_{1}$ versus $i_{3} \in[-1,1]$ for an increment of $\Delta i_{3}=0.001$; (b) bifurcation study with $x_{1}$ versus $s_{22} \in[1,1.3]$ for an increment of $\Delta s_{22}=0.001$; and (c) bifurcation study with $x_{1}$ versus $n \in[1,3.5]$ for an increment of $\Delta n=0.001$.

The alternative approach to determine whether a system is chaotic is to compute its Lyapunov exponents. The system is accepted as chaotic if it has at least one positive Lyapunov exponent, and all the trajectories are ultimately bounded [13]. By using the same parameter values in bifurcation studies, three Lyapunov exponent investigations are presented in Figure 3. As seen from the numerical results, the Lyapunov exponent spectrums with respect to $i_{3}, s_{22}$ and $n$, are consonant with corresponding phase and bifurcation diagrams. In our study, we developed an algorithm that estimates the dominant Lyapunov exponent of time series by monitoring orbital divergence of the proposed system in Equation (3). The Lyapunov exponent spectrums are calculated using the numerical methods described in [36]. In addition, a Poincaré section of the proposed system in $x_{1}-x_{3}$ domain can be seen in Figure 4 . 


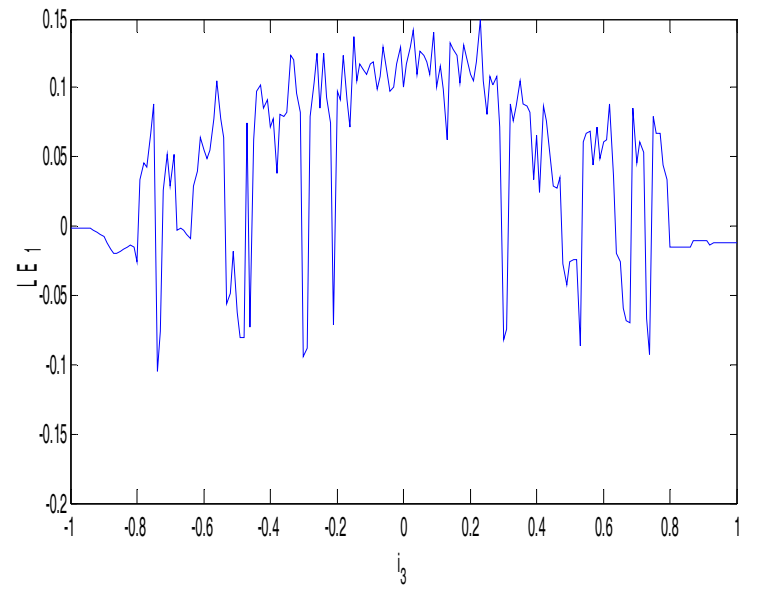

(a)

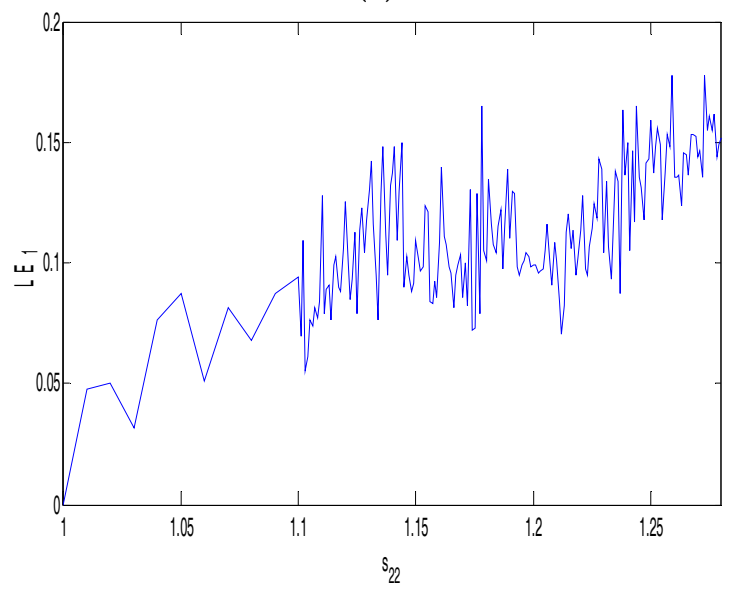

(c)

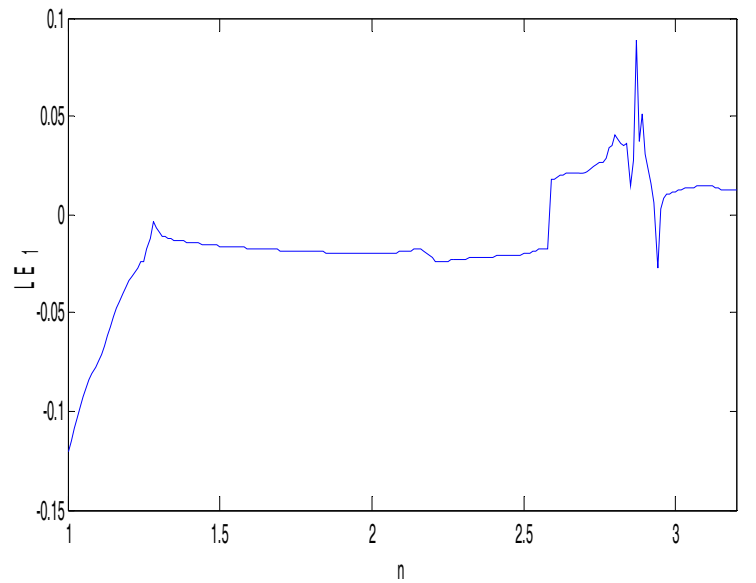

(e)

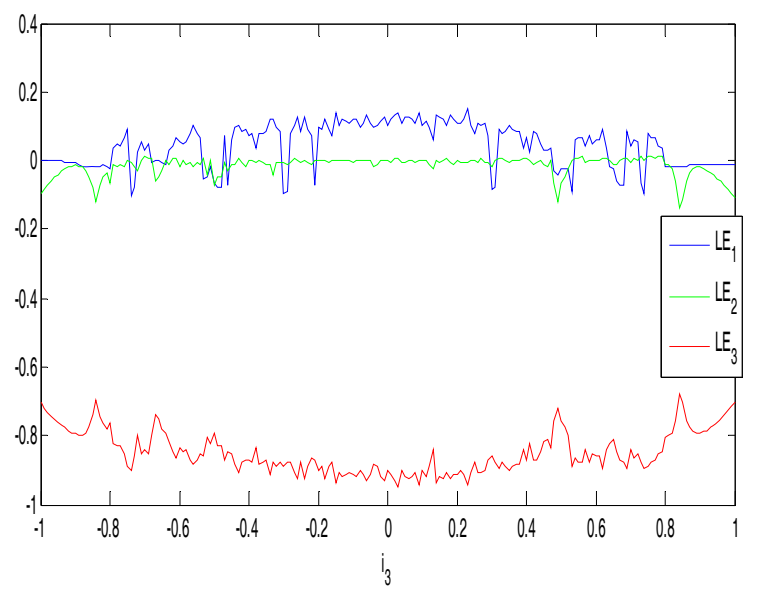

(b)

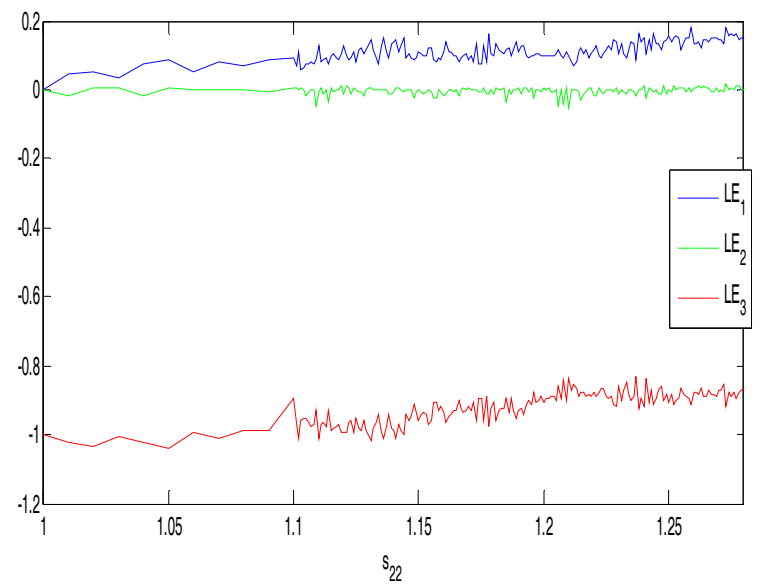

(d)

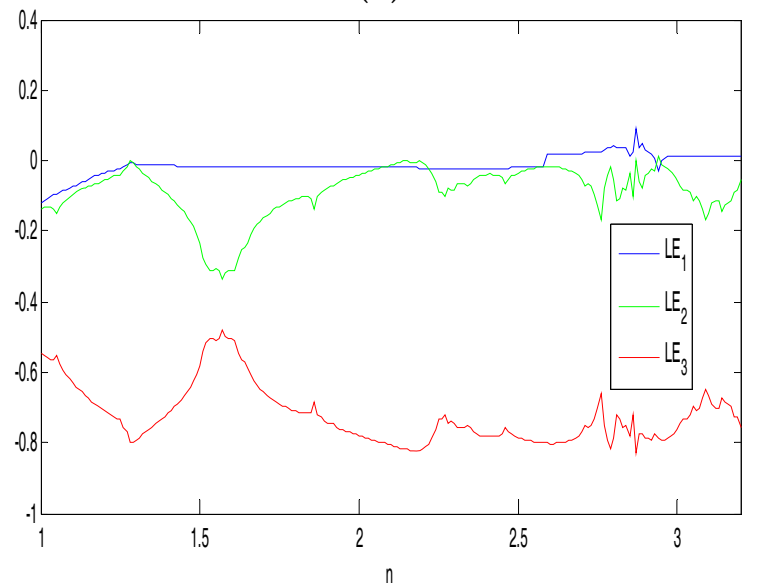

(f)

Figure 3. (a) Diagram of largest Lyapunov exponent for $i_{3} \in[-1,1]$; (b) Lyapunov exponents of the system for $i_{3} \in[-1,1]$; (c) diagram of largest Lyapunov exponent for $s_{22} \in[1,1.3]$; (d) Lyapunov exponents of the system for $s_{22} \in[1,1.3]$; (e) diagram of largest Lyapunov exponent for $n \in[1,3.5]$; and (f) Lyapunov exponents of the system for $n \in[1,3.5]$. 


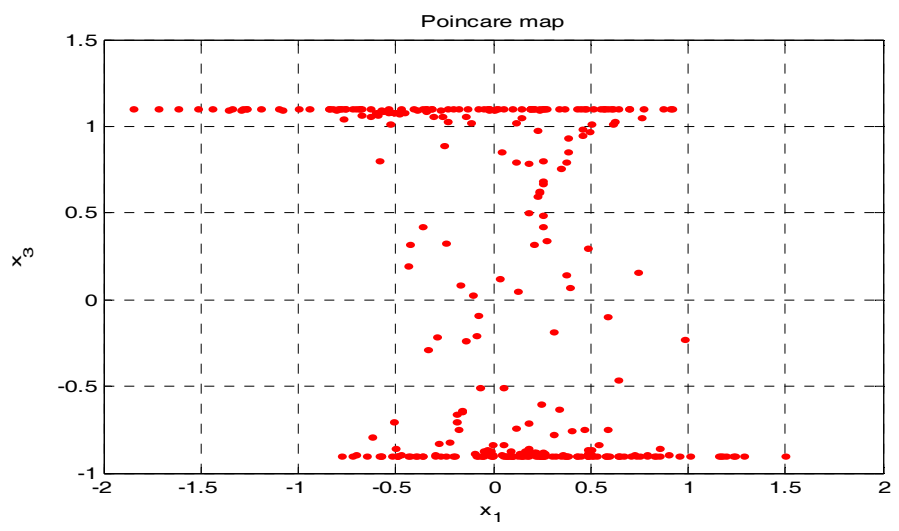

Figure 4. Poincaré mapping of the double-scroll attractor $\left(s_{11}=s_{12}=s_{13}=a_{32}=1 ; s_{21}=-1 ; s_{22}=1.2\right.$; $\left.n=10, i_{3}=0.1\right)$.

\section{Generating Multi-Scroll Chaotic Attractors}

In this section, we introduce approaches in generating multi-scroll chaotic attractors utilizing hyperbolic tangent function series in the proposed system. Firstly, hyperbolic tangent function series are added along $x_{3}$ to generate one direction (1-D) multi-scroll chaotic attractors. Then, to generate two directions (2-D) multi-scroll chaotic attractors, hyperbolic tangent function series are added to the system along $x_{1}-x_{3}$ directions and $x_{2}-x_{3}$ directions in two different ways. Finally, three direction (3-D) multi-scroll chaotic attractors are obtained by adding hyperbolic tangent function series to the system along $x_{1}-x_{2}-x_{3}$ directions in three different ways.

\subsection{Generating One Direction (1-D) Multi-Scroll Chaotic Attractors}

Consider the following system:

$$
\begin{gathered}
\dot{x}_{1}=x_{2}+x_{3} \\
\dot{x}_{2}=-x_{1}-x_{2}+s_{22} x_{2} \\
\dot{x}_{3}=-x_{3}-\tanh \left(n y_{2}\right)+i_{3}-\tanh \left(n y_{2}\right)-i_{3}
\end{gathered}
$$

where

$$
\begin{gathered}
s_{11}=s_{12}=s_{13}=a_{32}=1 ; s_{21}=-1 ; s_{22}=1.1, i_{3}=0.1, n=10 ; \text { and } \\
s_{23}=s_{31}=s_{32}=s_{33}=a_{11}=a_{12}=a_{13}=a_{21}=a_{22}=a_{23}=a_{31}=a_{33}=i_{1}=i_{2}=0 .
\end{gathered}
$$

The equilibrium points of Equation (10) exist in these three subspaces, defined as follows:

$$
\begin{gathered}
D_{1}=\left\{\left(x_{1}, x_{2}, x_{3}\right) \mid x_{2} \geq 1\right\}: P_{1}=\left(k_{1}, k_{2}, k_{3}\right), \\
D_{0}=\left\{\left(x_{1}, x_{2}, x_{3}\right)|| x_{2} \mid \leq 1\right\}: P_{0}=\left(l_{1}, l_{2}, l_{3}\right), \\
D_{2}=\left\{\left(x_{1}, x_{2}, x_{3}\right) \mid x_{2} \leq-1\right\}: P_{2}=\left(m_{1}, m_{2}, m_{3}\right)
\end{gathered}
$$

where

$$
\begin{gathered}
k_{1}=2 s_{22}-2, k_{2}=2, k_{3}=-2 m_{1}=2-2 s_{22}, m_{2}=-2, m_{3}=2 \\
l_{1}=0, l_{2}=0, l_{3}=0
\end{gathered}
$$

The equilibrium points $P_{1}, P_{0}$, and $P_{2}$ can be found from the Jacobian matrices.

$$
J_{1}=J_{2}=\left[\begin{array}{ccc}
0 & 1 & 1 \\
-1 & \left(s_{22}-1\right) & 0 \\
0 & 0 & -1
\end{array}\right] ; J_{0}=\left[\begin{array}{ccc}
0 & 1 & 1 \\
-1 & \left(s_{22}-1\right) & 0 \\
0 & 2 n & -1
\end{array}\right]
$$


Thus, the corresponding characteristic equations and eigenvalues take the following form:

$$
\left.\left.\begin{array}{c}
P_{1}(\lambda)=P_{2}(\lambda)=\lambda^{3}+\left(2-s_{22}\right) \lambda^{2}+\left(2-s_{22}\right) \lambda+1 \\
P_{0}(\lambda)=\lambda^{3}-\left(2-s_{22}\right) \lambda^{2}+\left(2-s_{22}\right) \lambda+2 n+1 \\
\lambda_{1}+\lambda_{2}+\lambda_{3}=\left(s_{22}-2\right)<0 \\
\lambda_{1} \lambda_{2} \lambda_{3}=-1<0
\end{array}\right\} \text { for } D_{1}, D_{2} \begin{array}{c}
\lambda_{1}+\lambda_{2}+\lambda_{3}=\left(s_{22}-2\right)<0 \\
\lambda_{1} \lambda_{2} \lambda_{3}=(-2 n-1)<0
\end{array}\right\} \text { for } D_{0}
$$

The initial conditions are taken as $(0.1,0.1,0.1)$ in this study. The eigenvalues for equilibrium point $(0,0,0) \in D_{0}$ are calculated from the matrix $J_{0}$ as $\lambda_{1}=-2.9731$ and $\lambda_{2,3}=1.0365 \pm 2.4472 i$, and the eigenvalues for equilibrium points $(-0.2,-2,2) \in D_{1}$ and $(0.2,2,-2) \in D_{2}$ are calculated from the matrix $J_{1,2}$ as $\lambda_{1}=-1$ and $\lambda_{2,3}=0.05 \pm 0.9987 i$.

Remark 1. System (10) can generate 1-D multi-scroll chaotic attractors via two basic strategies that can be summarized as follows:

- adding tanh functions in $x_{3}$ direction via $y_{2}$ nonlinear function; and

- $\quad$ parameterss $_{22}$ and $n$ satisfy condition given in Equation (15).

System (10) has one negative root and one pair of complex conjugate roots with positive real parts for all subspaces. Then, SC-CNN system becomes unstable and all equilibrium points $P$ are saddle points of index 2 . Figure $5 \mathrm{a}$, b shows $x_{1}-x_{2}-x_{3}$ plane projection of 2-double-scroll attractor with variable $x_{1}(\mathrm{t})$, respectively. Figure $5 \mathrm{c}, \mathrm{d}$ presents 4 -double-scroll attractor and time response of $x_{1}(\mathrm{t})$ for:

$$
\dot{x}_{3}=-x_{3}-\tanh \left(n y_{2}\right)+i_{3 a}-\tanh \left(n y_{2}\right)-i_{3 a}-\tanh \left(n y_{2}\right)+i_{3 b}-\tanh \left(n y_{2}\right)-i_{3 b}
$$

where $i_{3 a}=0.5$ and $i_{3 b}=1$.

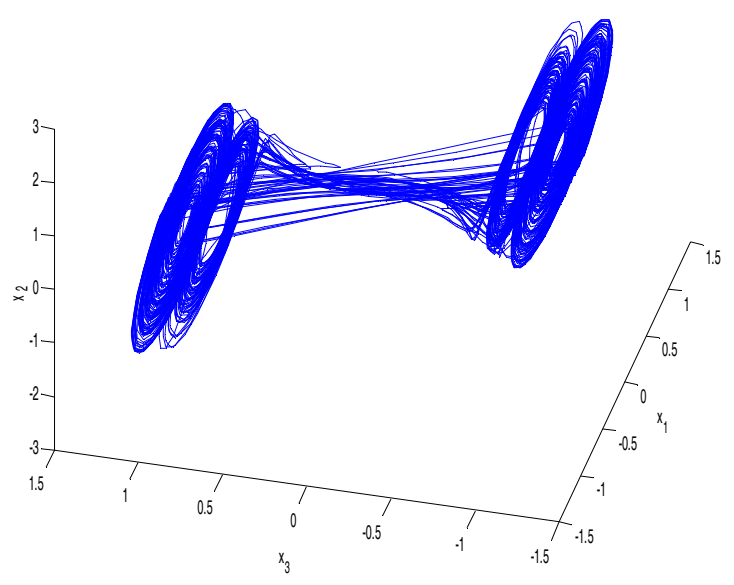

(a)

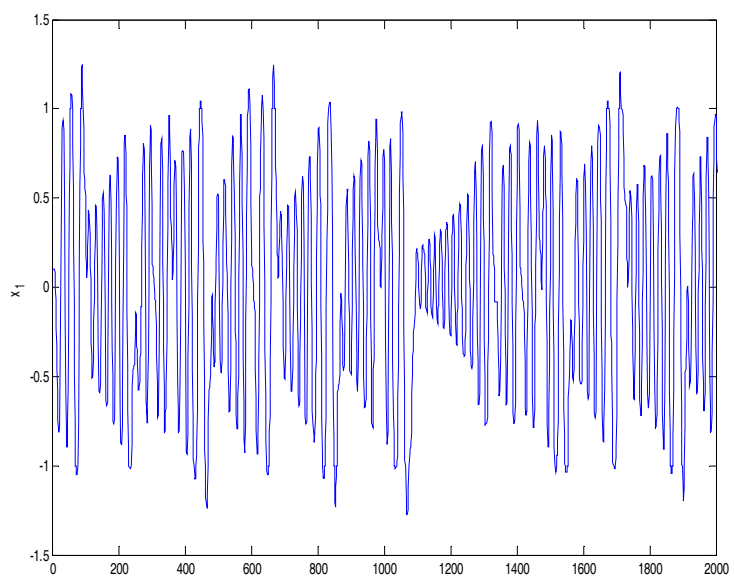

(b)

Figure 5. Cont. 


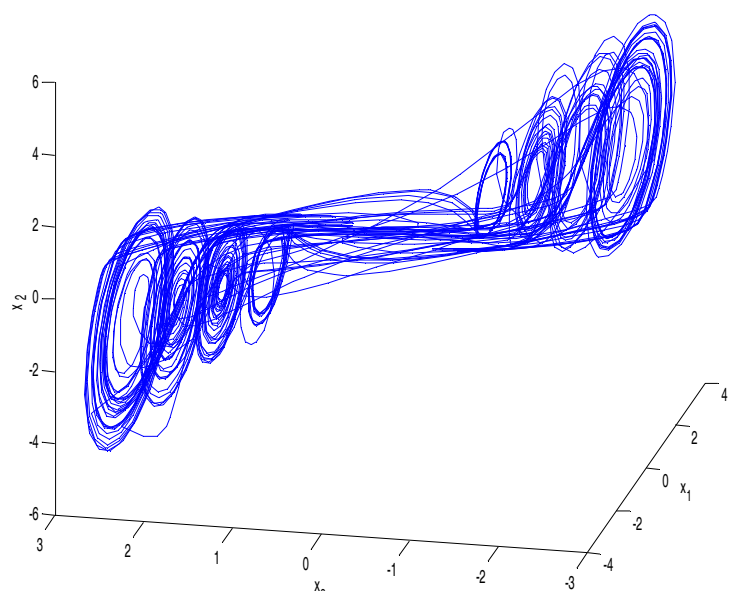

(c)

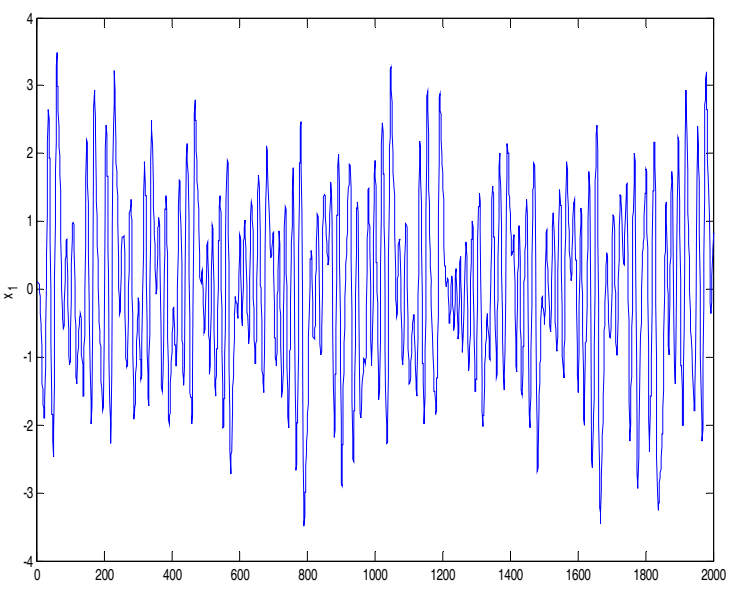

(d)

Figure 5. Numerical results of the 1-D multi-scroll SC-CNN Based system: (a) 2-double scrollin $x_{1}-x_{2}-x_{3}$ plane; (b) time response of $x_{1}(\mathrm{t})$ dynamic for 2-double scroll; (c) 4-double scrollin $x_{1}-x_{2}-x_{3}$ plane; and (d) time response of $x_{1}(\mathrm{t})$ dynamic for 4-double scroll.

\subsection{Generating Two Direction (2-D) Multi-Scroll Chaotic Attractors}

In this section, we introduce the ways of generating two-direction (2-D) multi-scroll chaotic attractors in SC-CNN based system. 2-D multi-scrolls can be generated by adding hyperbolic tangent function series to the SC-CNN system along $x_{1}-x_{3}$ directions and $x_{2}-x_{3}$ directions.

- In the first model, hyperbolic tangent function series are added along $x_{1}-x_{3}$ directions via $y_{3}$ and $y_{2}$ nonlinear functions, respectively.

- In the second model, hyperbolic tangent function series are added along $x_{1}-x_{3}$ directions by using $y_{2}$ nonlinear function, respectively.

- In the third model, hyperbolic tangent function series are injected to the system along $x_{2}-x_{3}$ directions via $y_{2}$ and $y_{1}$ nonlinear functions, respectively.

- In the fourth model, hyperbolic tangent function series are attached to the system along $x_{2}-x_{3}$ directions by using $y_{1}$ and $y_{2}$ nonlinear functions, respectively.

\subsection{1. $x_{1}-x_{3}$ Direction 2-D Multi-Scroll Attractors-I:}

Consider 2-D multi-scroll chaotic attractor generator:

$$
\begin{gathered}
\dot{x}_{1}=x_{2}+x_{3}+\tanh \left(n y_{3}\right)+i_{1} \\
\dot{x}_{2}=-x_{1}-x_{2}+s_{22} x_{2} \\
\dot{x}_{3}=-x_{3}-\tanh \left(n y_{2}\right)+i_{3} \\
s_{11}=s_{12}=s_{13}=a_{11}=a_{32}=1 ; s_{21}=-1 ; s_{22}=1.1, i_{1}=i_{3}=0.1, n=10 ; \text { and } \\
s_{23}=s_{31}=s_{32}=s_{33}=a_{12}=a_{13}=a_{21}=a_{22}=a_{23}=a_{31}=a_{33}=i_{2}=0 .
\end{gathered}
$$

The equilibrium points of Equation (17) exist in these three subspaces, defined as follows:

$$
\begin{gathered}
D_{4}=\left\{\left(x_{1}, x_{2}, x_{3}\right) \mid x_{2}, x_{3} \geq 1\right\}: P_{4}=\left(k_{4}, k_{5}, k_{6}\right), \\
D_{3}=\left\{\left(x_{1}, x_{2}, x_{3}\right)|| x_{2}|,| x_{3} \mid \leq 1\right\}: P_{3}=\left(l_{4}, l_{5}, l_{6}\right), \\
D_{5}=\left\{\left(x_{1}, x_{2}, x_{3}\right) \mid x_{2}, x_{3} \leq-1\right\}: P_{5}=\left(m_{4}, m_{5}, m_{6}\right) \\
k_{4}=m_{7}=-\left(i_{1}+i_{3}\right)\left(s_{22}-1\right), k_{5}=m_{8}=-1-i_{3}, k_{6}=m_{9}=i_{3}-1 \\
l_{4}=\frac{-\left(i_{2}+i_{3}+i_{2} n-i_{3} s_{22}\right)}{(n+1)(n-1)}, l_{5}=\frac{-i_{3}}{(n+1)}, l_{6}=\frac{i_{3}}{(n+1)}
\end{gathered}
$$


The equilibrium points $P_{6}, P_{7}$, and $P_{8}$ can be found from the Jacobian matrices.

$$
J_{4}=J_{5}=\left[\begin{array}{ccc}
0 & 1 & 1 \\
-1 & \left(s_{22}-1\right) & 0 \\
0 & 0 & -1
\end{array}\right], J_{3}=\left[\begin{array}{ccc}
0 & 1 & a_{32} n+1 \\
-1 & \left(s_{22}-1\right) & 0 \\
0 & -a_{32} n & -1
\end{array}\right]
$$

The corresponding characteristic equation is:

$$
\begin{gathered}
P_{4}(\lambda)=P_{5}(\lambda)=\lambda^{3}+\left(2-s_{22}\right) \lambda^{2}+\left(2-s_{22}\right) \lambda+1 \\
P_{3}(\lambda)=\lambda^{3}+\left(2-s_{22}\right) \lambda^{2}-\left(2-s_{22}\right) \lambda+a_{32} a_{13} n^{2}-a_{32} n+1 \\
\left.\lambda_{1}+\lambda_{2}+\lambda_{3}=\left(\begin{array}{c}
\left.s_{22}-2\right)<0 \\
\lambda_{1} \lambda_{2} \lambda_{3}=-1<0
\end{array}\right\} \text { for } D_{4}, D_{5} \begin{array}{c}
\lambda_{1}+\lambda_{2}+\lambda_{3}=\left(s_{22}-2\right)<0 \\
\lambda_{1} \lambda_{2} \lambda_{3}=\left(a_{32} a_{13} n^{2}+a_{32} n-1\right)>0
\end{array}\right\} \text { for } D_{3}
\end{gathered}
$$

The system can produce chaotic behaviors for most of the initial conditions and those are taken as $(0.1,0.1,0.1)$ in this model. The eigenvalues for equilibrium point $(-0.02,-0.2,-0.9) \in D_{3}$ are calculated from the matrix $J_{3}$ as $\lambda_{1}=-5.0591$ and $\lambda_{2,3}=2.0795 \pm 4.1972 i$, and the eigenvalues for equilibrium points $(-0.02,-0.2,-0.9) \in D_{4}$ and $(-0.02,-0.2,1.1) \in D_{5}$ are calculated from the matrix $J_{4,5}$ as $\lambda_{1}=-1$ and $\lambda_{2,3}=0.05 \pm 0.987 i$.

Remark 2. The strategies which can generate 2-D multi-scroll chaotic attractors in System (17):

- adding tanh functions in $x_{1}$ via $y_{3}$ nonlinear function, and $x_{3}$ direction via $y_{2}$ nonlinear function; and

- parameterss $_{22}, n, a_{13}$, anda $a_{32}$ satisfy condition Equation (22).

System (17) has one negative root and one pair of complex conjugate roots with positive real parts for all subspaces, and SC-CNN System (17) becomes unstable and equilibrium $P$ is a saddle point index 2. Figure 6a shows $x_{1}-x_{2}-x_{3}$ plane projection of 2-double-scroll attractor. Figure $6 \mathrm{~b}$ presents time domain responses of $x_{2}(\mathrm{t})$, Figure $6 \mathrm{c}, \mathrm{d}$ presents 12-double-scroll attractor and time response of $x_{1}(\mathrm{t})$ for:

$$
\begin{gathered}
\dot{x}_{1}=x_{2}+x_{3}+\tanh \left(n y_{3}\right)+i_{1 a}+\tanh \left(n y_{3}\right)-i_{1 a}+ \\
\tanh \left(n y_{3}\right)+i_{1 b}+\tanh \left(n y_{3}\right)-i_{1 b}+ \\
\tanh \left(n y_{3}\right)+i_{1 c}+\tanh \left(n y_{3}\right)-i_{1 c} \\
\dot{x}_{2}=-x_{1}-x_{2}+s_{22} x_{2} \\
\dot{x}_{3}=-x_{3}-\tanh \left(n y_{2}\right)+i_{3 a}-\tanh \left(n y_{2}\right)-i_{3 a}- \\
\tanh \left(n y_{2}\right)+i_{3 b}-\tanh \left(n y_{2}\right)-i_{3 b}- \\
\tanh \left(n y_{2}\right)+i_{3 c}-\tanh \left(n y_{2}\right)-i_{3 c}
\end{gathered}
$$

where $i_{1 a}=i_{3 a}=0.1, i_{1 b}=i_{3 b}=0.2$, and $i_{1 c}=i_{3 c}=0.3$. 


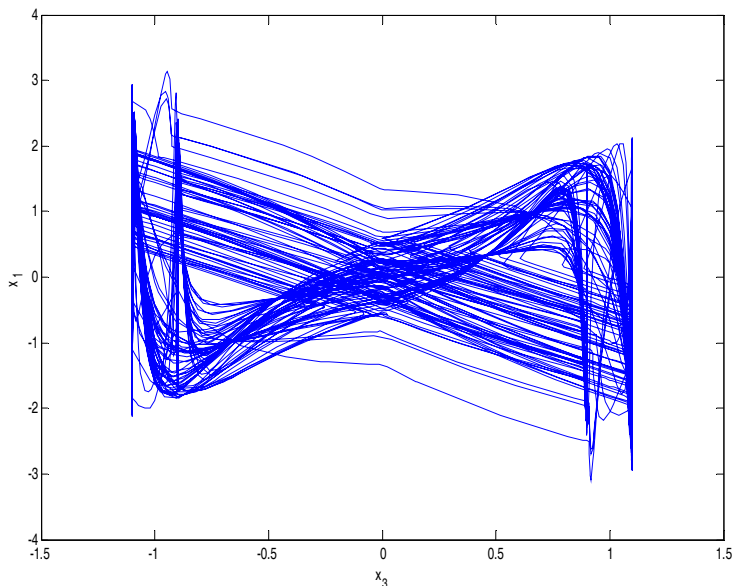

(a)

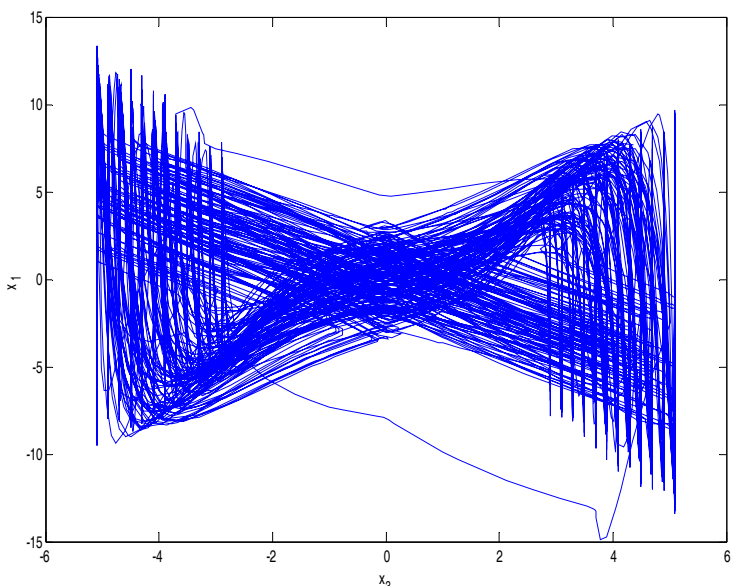

(c)

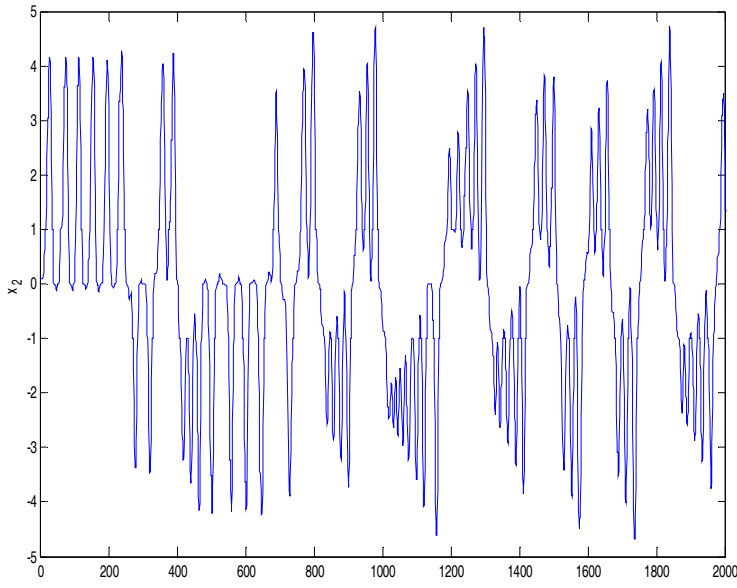

(b)

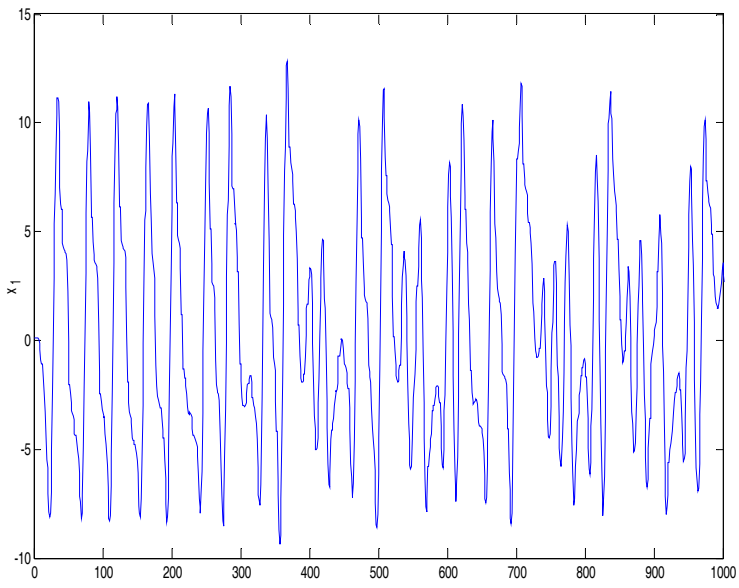

(d)

Figure 6. Numerical results of the $x_{1}-x_{3}$ direction2-D multi-scroll SC-CNN based system-I: (a) 2-double scrollin $x_{1}-x_{2}-x_{3}$ plane; (b) variable $x_{1}(\mathrm{t}) ;(\mathbf{c}) 12$-double scrollin $x_{1}-x_{3}$ plane; and (d) variable $x_{1}(\mathrm{t})$.

\subsection{2. $x_{1}-x_{3}$ Direction 2-D Multi-Scroll Attractor-II:}

$$
\begin{gathered}
\dot{x}_{1}=x_{2}+x_{3}-\tanh \left(n y_{2}\right)+i_{1} \\
\dot{x}_{2}=-x_{1}-x_{2}+s_{22} x_{2} \\
\dot{x}_{3}=-x_{3}-\tanh \left(n y_{2}\right)+i_{3} \\
s_{11}=s_{12}=s_{13}=a_{12}=a_{32}=1 ; s_{21}=-1 ; s_{22}=1.1, i_{1}=i_{3}=0.1, n=10 ; \text { and } \\
s_{23}=s_{31}=s_{32}=s_{33}=a_{11}=a_{13}=a_{21}=a_{22}=a_{23}=a_{31}=a_{33}=i_{2}=0 .
\end{gathered}
$$

Three subspaces can be defined as follows:

$$
\begin{gathered}
D_{7}=\left\{\left(x_{1}, x_{2}, x_{3}\right) \mid x_{2} \geq 1\right\}: P_{7}=\left(k_{7}, k_{8}, k_{9}\right), \\
D_{6}=\left\{\left(x_{1}, x_{2}, x_{3}\right)|| x_{2} \mid \leq 1\right\}: P_{6}=\left(l_{7}, l_{8}, l_{9}\right), \\
D_{8}=\left\{\left(x_{1}, x_{2}, x_{3}\right) \mid x_{2} \leq-1\right\}: P_{8}=\left(m_{7}, m_{8}, m_{9}\right) \\
k_{7}=-\left(s_{22}-1\right)\left(i_{1}+i_{3}-2\right), k_{8}=2-i_{3}-i_{1}, k_{9}=i_{3}-1 \\
m_{7}=-\left(s_{22}-1\right)\left(i_{1}+i_{3}+2\right), m_{8}=2-i_{3}-i_{1}, m_{9}=i_{3}+1, \\
l_{7}=\frac{\left[\left(s_{22}-1\right)\left(i_{1}+i_{3}-a_{32} i_{1} n\right)\right]}{\left(a_{12} n+1\right)\left(a_{32} n-1\right)}, l_{8}=\frac{\left(i_{1}+i_{3}-a_{32} i_{1} n\right)}{\left(a_{12} n+1\right)\left(a_{32} n-1\right)}, l_{9}=\frac{-i_{3}}{\left(a_{32} n-1\right)}
\end{gathered}
$$


The equilibrium points $P_{12}, P_{13}$, and $P_{14}$ can be found from the Jacobian matrices.

$$
J_{7}=J_{8}=\left[\begin{array}{ccc}
0 & 1 & 1 \\
-1 & \left(s_{22}-1\right) & 0 \\
0 & 0 & -1
\end{array}\right] ; J_{6}=\left[\begin{array}{ccc}
0 & a_{12} n+1 & 1 \\
-1 & \left(s_{22}-1\right) & 0 \\
0 & 0 & a_{32} n-1
\end{array}\right]
$$

The corresponding characteristic equation is:

$$
\begin{aligned}
& P_{7}(\lambda)=P_{8}(\lambda)=\lambda^{3}+\left(2-s_{22}\right) \lambda^{2}+\left(2-s_{22}\right) \lambda+1 \\
& P_{6}(\lambda)=\lambda^{3}-\left(s_{22}+a_{32} n-2\right) \lambda^{2}-\left(a_{12} n-s_{22}-a_{32} n+a_{32} n s_{22}+2\right) \lambda-a_{12} a_{32} n^{2} \\
& +a_{12} n-a_{32} n+1 \\
& \left.\left.\begin{array}{rl}
\lambda_{1}+\lambda_{2}+\lambda_{3} & =\left(s_{22}-2\right)<0 \\
\lambda_{1} \lambda_{2} \lambda_{3} & =-1<0
\end{array}\right\} \text { for } D_{7}, D_{8} \begin{array}{r}
\lambda_{1}+\lambda_{2}+\lambda_{3}=\left(s_{22}+a_{32} n-2\right)>0 \\
\lambda_{1} \lambda_{2} \lambda_{3}=\left(a_{12} n+1\right)\left(a_{32} n-1\right)>0
\end{array}\right\} \text { for } D_{6}
\end{aligned}
$$

Remark 3. System (24) can generate 2-D multi-scroll chaotic attractors:

- $\quad$ adding tanh functions in $x_{1}$ and $x_{3}$ direction via $y_{2}$ nonlinear function; and

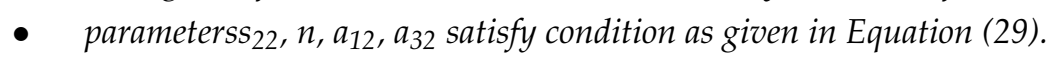

The system can produce chaotic behaviors for most of the initial conditions and those are taken as $(0.1,0.1,0.1)$ in this paper. The eigenvalues for equilibrium point $(0,-0.01,-0.01) \in D_{6}$ are calculated from the matrix $J_{6}$ as $\lambda_{1}=9$ and $\lambda_{2,3}=0.05 \pm 3.3162 i$, and the eigenvalues for equilibrium points $(0.18$, $1.8,-0.9) \in D_{7}$ and $(-0.22,-2.2,1.1) \in D_{8}$ are calculated from the matrix $J_{7,8}$ as $\lambda_{1}=-1$ and $\lambda_{2,3}=0.05$ $\pm 0.9987 i$. System (24) has one positive root and one pair of complex conjugate roots with positive real parts for $D_{6}$ subspace, and SC-CNN System (24) becomes unstable and equilibria $P_{6}$ is afocus node. On the other hand, System (24) has one negative root and one pair of complex conjugate roots with positive real parts for $D_{7,8}$ subspaces. Thus, SC-CNN System (24) becomes unstable and equilibrium points $P_{7,8}$ are saddle point index 2 . Figure 7a shows $x_{1}-x_{3}$ plane projection of 2-double-scroll attractor. Figure $6 \mathrm{~b}$ presents time domain responses of $x_{2}(\mathrm{t})$, Figure $6 \mathrm{c}, \mathrm{d}$ presents 12-double-scroll attractor and time response of $x_{1}(\mathrm{t})$ for:

$$
\begin{gathered}
\dot{x}_{1}=x_{2}+x_{3}-\tanh \left(n y_{2}\right)+i_{1 a}-\tanh \left(n y_{2}\right)-i_{1 a}- \\
\tanh \left(n y_{2}\right)+i_{1 b}-\tanh \left(n y_{2}\right)-i_{1 b}- \\
\tanh \left(n y_{2}\right)+i_{1 c}-\tanh \left(n y_{2}\right)-i_{1 c} \\
\dot{x}_{2}=-x_{1}-x_{2}+s_{22} x_{2} \\
\dot{x}_{3}=-x_{3}-\tanh \left(n y_{2}\right)+i_{3 a}-\tanh \left(n y_{2}\right)-i_{3 a}- \\
\tanh \left(n y_{2}\right)+i_{3 b}-\tanh \left(n y_{2}\right)-i_{3 b}- \\
\tanh \left(n y_{2}\right)+i_{3 c}-\tanh \left(n y_{2}\right)-i_{3 c}
\end{gathered}
$$

where $i_{1 a}=i_{3 a}=0.1, i_{1 b}=i_{3 b}=0.2$, and $i_{1 c}=i_{3 c}=0.3$. 


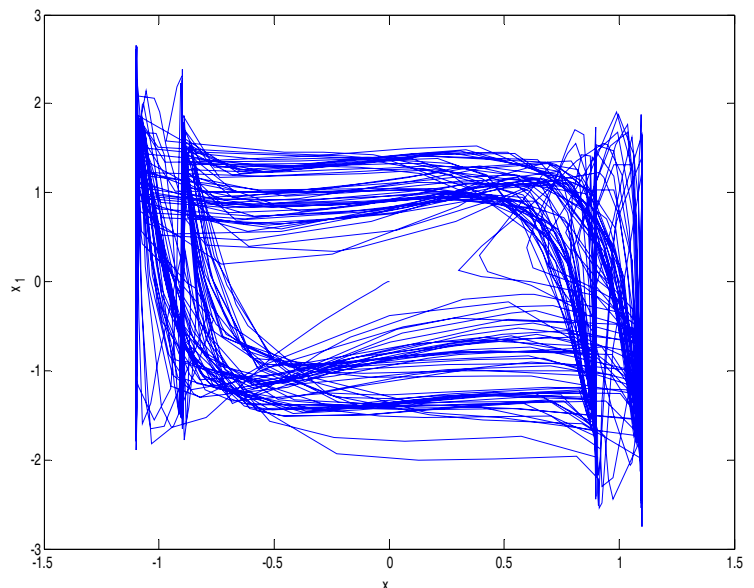

(a)

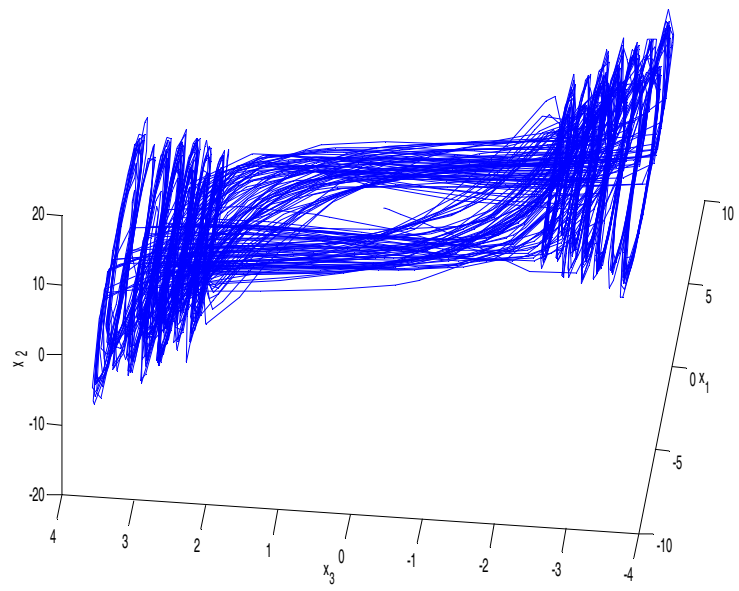

(c)

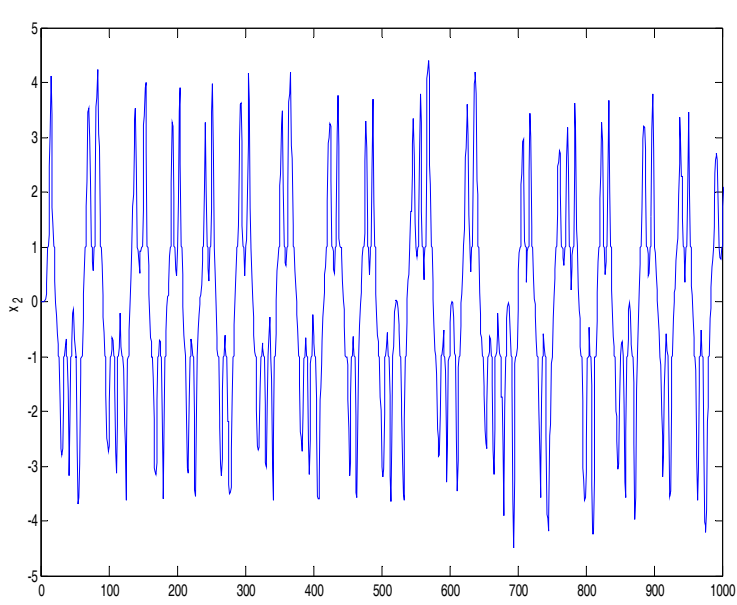

(b)

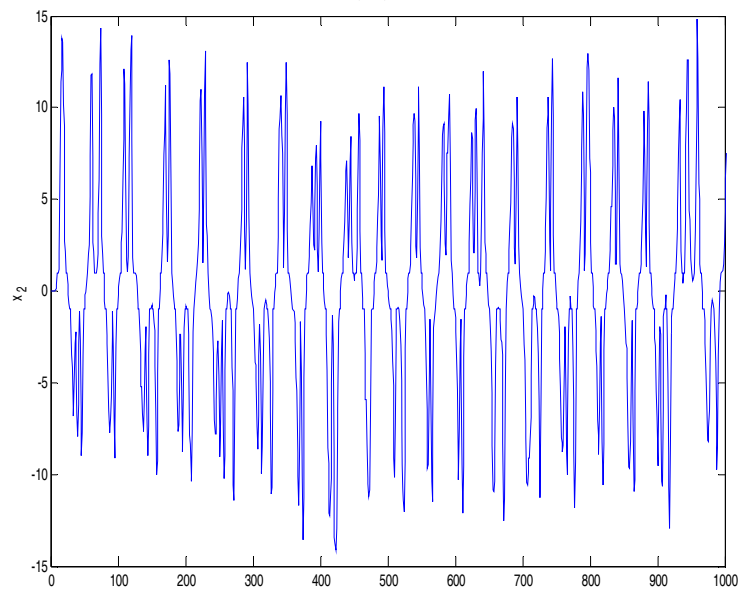

(d)

Figure 7. Numerical results of the $x_{1}-x_{3}$ direction2-D multi-scroll SC-CNN based system-II: (a) 2-double scrollin $x_{1}-x_{2}-x_{3}$ plane; (b) variable $x_{1}(\mathrm{t}) ;(\mathbf{c}) 7$-double scrollin $x_{1}-x_{2}-x_{3}$ plane; and (d) variable $x_{2}(\mathrm{t})$.

4.2.3. $x_{2}-x_{3}$ Direction 2-D Multi-Scroll Attractors-I:

$$
\begin{gathered}
\dot{x}_{1}=x_{2}+x_{3} \\
\dot{x}_{2}=-x_{1}-x_{2}+s_{22} x_{2}-\tanh \left(n y_{2}\right)+i_{2} \\
\dot{x}_{3}=-x_{3}+\tanh \left(n y_{1}\right)+i_{3} \\
s_{11}=s_{12}=s_{13}=a_{31}=a_{22}=1 ; s_{21}=-1 ; s_{22}=1.05, i_{2}=i_{3}=0.1, n=10 ; \\
s_{23}=s_{31}=s_{32}=s_{33}=a_{11}=a_{12}=a_{13}=a_{23}=a_{32}=a_{33}=i_{1}=0 ;
\end{gathered}
$$

The equilibrium points of Equation (31) exist in these three subspaces defined as follows:

$$
\begin{gathered}
D_{10}=\left\{\left(x_{1}, x_{2}, x_{3}\right) \mid x_{1}, x_{2} \geq 1\right\}: P_{10}=\left(k_{10}, k_{11}, k_{12}\right) \\
D_{9}=\left\{\left(x_{1}, x_{2}, x_{3}\right)|| x_{1}|,| x_{2} \mid \leq 1\right\}: P_{9}=\left(l_{10}, l_{11}, l_{12}\right), \\
D_{11}=\left\{\left(x_{1}, x_{2}, x_{3}\right) \mid x_{1}, x_{2} \leq-1\right\}: P_{11}=\left(m_{10}, m_{11}, m_{12}\right) \\
k_{10}=i_{2}+i_{3}-s_{22}-i_{3} s_{22}, k_{11}=-1-i_{3}, k_{12}=i_{3}+1 \\
m_{10}=i_{2}+i_{3}+s_{22}-i_{3} s_{22}, m_{11}=1-i_{3}, m_{12}=i_{3}-1
\end{gathered}
$$




$$
\begin{aligned}
& l_{10}=\frac{-\left(i_{2}+i_{3}-i_{3} s_{22}-a_{22} i_{3} n\right)}{\left(a_{31} n_{22}-a_{31} 1+a_{33} a_{22} n^{2}+1\right)}, \\
& l_{11}=\frac{-\left(i_{3}+a_{31} i_{2} n\right)}{\left(a_{31} n s_{22}-a_{31} n+a_{31} a_{22} n^{2}+1\right)}, \\
& l_{12}=\frac{\left(i_{3}+a_{31} 1_{2} n\right)}{\left(a_{31} n s_{22}-a_{31} n+a_{31} a_{22} n^{2}+1\right)}
\end{aligned}
$$

The equilibrium points $P_{9}, P_{10}$, and $P_{11}$ can be found from the Jacobian matrices.

$$
J_{10}=J_{11}=\left[\begin{array}{ccc}
0 & 1 & 1 \\
-1 & \left(s_{22}-1\right) & 0 \\
0 & 0 & -1
\end{array}\right], J_{9}=\left[\begin{array}{ccc}
0 & 1 & 1 \\
-1 & \left(s_{22}+a_{22} n-1\right) & 0 \\
a_{31} n & 0 & -1
\end{array}\right]
$$

The corresponding characteristic equation is:

$$
\begin{aligned}
& P_{10}(\lambda)=P_{11}(\lambda)=\lambda^{3}+\left(2-s_{22}\right) \lambda^{2}+\left(2-s_{22}\right) \lambda+1 \\
& P_{9}(\lambda)=\lambda^{3}-\left(s_{22}+a_{22} n-2\right) \lambda^{2}-\left(s_{22}+a_{31} n+a_{22} n-2\right) \lambda+a_{31} a_{22} n^{2}+a_{31} n s_{22}-a_{31} n+1 \\
& \left.\begin{array}{rl}
\lambda_{1}+\lambda_{2}+\lambda_{3} & =\left(s_{22}-2\right)<0 \\
\lambda_{1} \lambda_{2} \lambda_{3} & =-1<0
\end{array}\right\} \text { for } D_{10}, D_{11} \\
& \left.\begin{array}{l}
\lambda_{1}+\lambda_{2}+\lambda_{3}=\left(s_{22}+a_{22} n-2\right)>0 \\
\lambda_{3}=\left[\left(a_{31}-a_{31} s_{22}\right) n-a_{31} a_{22} n^{2}-1\right]<0
\end{array}\right\} \text { for } D_{9}
\end{aligned}
$$

Remark 4. System (31) can generate 2-D multi-scroll chaotic attractors via two basic strategies that can be summarized as follows:

- adding tanh functions in $x_{2}$ direction via $y_{2}$ nonlinear function and in $x_{3}$ direction via $y_{1}$ nonlinear function; and

- $\quad$ parameterss $_{22}, n, a_{22}$, and $a_{31}$ satisfy condition as given in Equation (36).

Initial conditions are taken as $(0.1,0.1,0.1)$ in this paper. The eigenvalues for equilibrium point $(-0.06,-0.05,0.05) \in D_{9}$ are calculated from the matrix $J_{9}$ as $\lambda_{1}=-1.2469$ and $\lambda_{2,3}=0.7234 \pm 1.0396 i$, and the eigenvalues for equilibrium points $(-0.9550,-1.1,1.1) \in D_{10}$ and $(1.1450,0.9,-0.9) \in D_{11}$ are calculated from the matrix $J_{10,11}$ as $\lambda_{1}=-1$ and $\lambda_{2,3}=0.0250 \pm 0.9997 i$. System (29) has one negative root and one pair of complex conjugate roots with positive real parts for all subspaces. Then, System (31) is unstable and all equilibrium points $P$ are saddle points of index 2. Figure 8 a shows $x_{1}-x_{2}-x_{3}$ plane projection of 2-double-scroll attractor. Figure $8 \mathrm{~b}$ presents time domain responses of $x_{1}(\mathrm{t})$, Figure $8 \mathrm{c}, \mathrm{d}$ presents 4-double-scroll attractor and time response of $x_{2}(\mathrm{t})$ for:

$$
\begin{gathered}
\dot{x}_{1}=x_{2}+x_{3} \\
\dot{x}_{2}=-x_{1}-x_{2}+s_{22} x_{2}-\tanh \left(n y_{2}\right)+i_{2 a}-\tanh \left(n y_{2}\right)-i_{2 a}- \\
\tanh \left(n y_{2}\right)+i_{2 b}-\tanh \left(n y_{2}\right)-i_{2 b} \\
\dot{x}_{3}=-x_{3}+\tanh \left(n y_{1}\right)+i_{3 a}+\tanh \left(n y_{1}\right)-i_{3 a}+ \\
\tanh \left(n y_{1}\right)+i_{3 b}+\tanh \left(n y_{1}\right)-i_{3 b}
\end{gathered}
$$

where $i_{2 a}=i_{3 a}=0.1$, and $i_{2 b}=i_{3 b}=0.2$. 


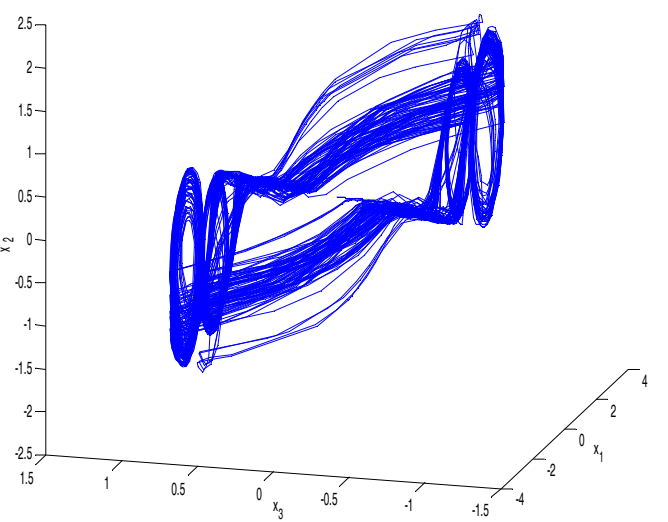

(a)

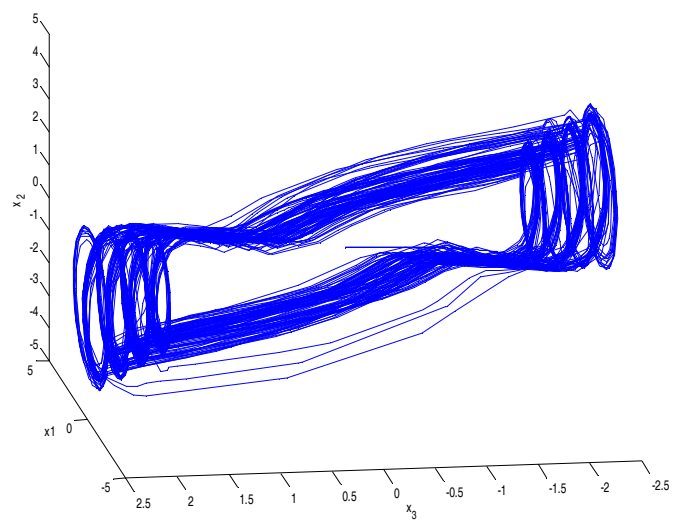

(c)

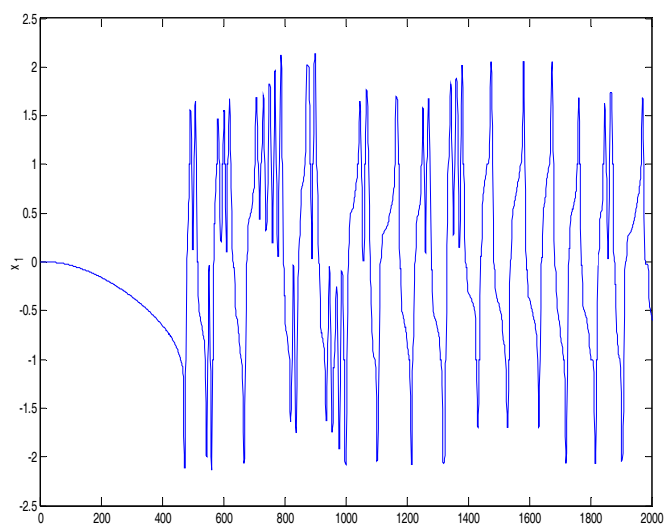

(b)

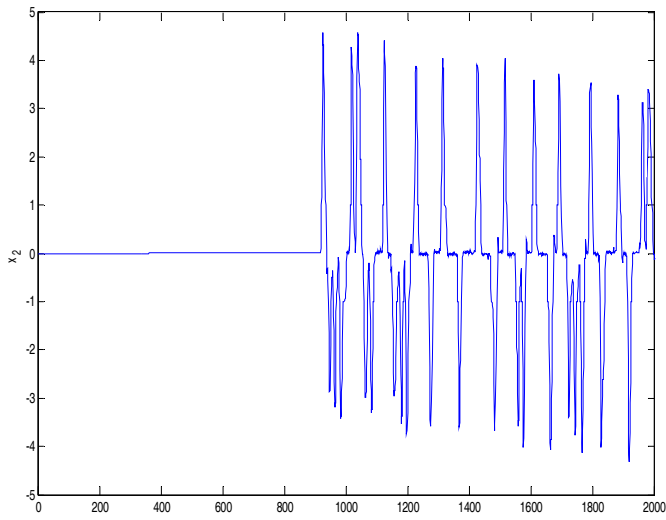

(d)

Figure 8. Numerical results of the $x_{2}-x_{3}$ direction 2-D multi-scroll SC-CNN based system-I: (a) 2-doublescroll in $x_{1}-x_{2}-x_{3}$ plane; (b) variable $x_{1}(\mathrm{t})$; (c) 4-double scrollin $x_{1}-x_{2}-x_{3}$ plane; and (d) variable $x_{2}(\mathrm{t})$.

\subsection{4. $x_{2}-x_{3}$ Direction 2-D Multi-Scroll Attractors-II:}

Consider the dynamical system:

$$
\begin{gathered}
\dot{x}_{1}=x_{2}+x_{3} \\
\dot{x}_{2}=-x_{1}-x_{2}+s_{22} x_{2}+\tanh \left(n y_{1}\right)+i_{2} \\
\dot{x}_{3}=-x_{3}-\tanh \left(n y_{2}\right)+i_{3} \\
s_{11}=s_{12}=s_{13}=a_{21}=a_{32}=1 ; s_{21}=-1 ; s_{22}=1.05, i_{2}=i_{3}=0.1, n=10 ; \\
s_{23}=s_{31}=s_{32}=s_{33}=a_{11}=a_{12}=a_{13}=a_{22}=a_{23}=a_{31}=a_{33}=i_{1}=0 ;
\end{gathered}
$$

Three subspaces can be defined as follows:

$$
\begin{gathered}
D_{13}=\left\{\left(x_{1}, x_{2}, x_{3}\right) \mid x_{1}, x_{2} \geq 1\right\}: P_{13}=\left(k_{13}, k_{14}, k_{15}\right) \\
D_{12}=\left\{\left(x_{1}, x_{2}, x_{3}\right)|| x_{1}|,| x_{2} \mid \leq 1\right\}: P_{12}=\left(l_{13}, l_{14}, l_{15}\right), \\
D_{14}=\left\{\left(x_{1}, x_{2}, x_{3}\right) \mid x_{1}, x_{2} \leq-1\right\}: P_{14}=\left(m_{13}, m_{14}, m_{15}\right) \\
k_{13}=i_{2}+i_{3}+s_{22}-i_{3} s_{22}, \quad k_{14}=1-i_{3}, \quad k_{15}=i_{3}-1 \\
m_{13}=i_{2}+i_{3}-s_{22}-i_{3} s_{22}, \quad m_{14}=-1-i_{3}, m_{15}=i_{3}+1 \\
l_{13}=\frac{-\left(i_{2}+i_{3}+i_{2} n-i_{3} s_{22}\right)}{(n+1)(n-1)}, \quad l_{14}=\frac{-i_{3}}{(n+1)}, \quad l_{15}=\frac{i_{3}}{(n+1)}
\end{gathered}
$$


The equilibrium points $P_{12}, P_{13}$, and $P_{14}$ can be found from the Jacobian matrices.

$$
J_{13}=J_{14}=\left[\begin{array}{ccc}
0 & 1 & 1 \\
-1 & \left(s_{22}-1\right) & 0 \\
0 & 0 & -1
\end{array}\right], J_{12}=\left[\begin{array}{ccc}
0 & 1 & 1 \\
n-1 & \left(s_{22}-1\right) & 0 \\
0 & n & -1
\end{array}\right]
$$

The corresponding characteristic equation is:

$$
\begin{gathered}
P_{13}(\lambda)=P_{14}(\lambda)=\lambda^{3}+\left(2-s_{22}\right) \lambda^{2}+\left(2-s_{22}\right) \lambda+1 \\
P_{12}(\lambda)=\lambda^{3}-\left(s_{22}-2\right) \lambda^{2}-\left(n+s_{22}-2\right) \lambda+1-n^{2} \\
\left.\begin{array}{c}
\lambda_{1}+ \\
\lambda_{2}+\lambda_{3}=\left(s_{22}-2\right)<0 \\
\lambda_{1} \lambda_{2} \lambda_{3}=-1<0 \\
\lambda_{1}+\lambda_{2}+\lambda_{3}=\left(s_{22}-2\right)<0 \\
\lambda_{1} \lambda_{2} \lambda_{3}=\left(n^{2}-1\right)>0
\end{array}\right\} \text { for } D_{13}, D_{14}
\end{gathered}
$$

The system can produce chaotic behaviors for most of the initial conditions and those are taken as $(0.1,0.1,0.1)$ in this paper. The eigenvalues for equilibrium point $(-0.011,-0.09,0.09) \in D_{12}$ are calculated from the matrix $J_{12}$ as $\lambda_{1}=4.9396$ and $\lambda_{2,3}=-2.9448 \pm 3.3777 i$, and the eigenvalues for equilibrium points $(1.145,0.9,-0.9) \in D_{13}$ and $(-0.9550,-1.1,1.1) \in D_{14}$ are calculated from the matrix $J_{13,14}$ as $\lambda_{1}=-1$ and $\lambda_{2,3}=0.0250 \pm 0.9997 i$. System (38) has one positive root and one pair of complex conjugate roots with negative real parts for $D_{12}$ subspace, and SC-CNN System (38) becomes unstable and equilibria $P_{12}$ is a saddle point index 1 .

On the other hand, System (38) has one negative root and one pair of complex conjugate roots with positive real parts for $D_{13,14}$ subspaces. Thus, SC-CNN System (38) becomes unstable and equilibrium points $P_{13,14}$ are saddle point index 2 . Figure 9 shows $x_{1}-x_{2}-x_{3}$ plane projection of 4-double-scroll attractor with $x_{1}(\mathrm{t}), x_{2}(\mathrm{t})$ and $x_{3}(\mathrm{t})$ variables, respectively.

Remark 5. There are two basic mechanisms to generate 2-D multi-scroll chaotic attractors in System (38):

- adding tanh functions in $x_{1}$ direction via $y_{1}$ nonlinear function and in $x_{3}$ direction via $y_{2}$ nonlinear function; and

- $\quad$ parameter $\operatorname{ss}_{22}$ and $n$ satisfy condition as given in Equation (43).

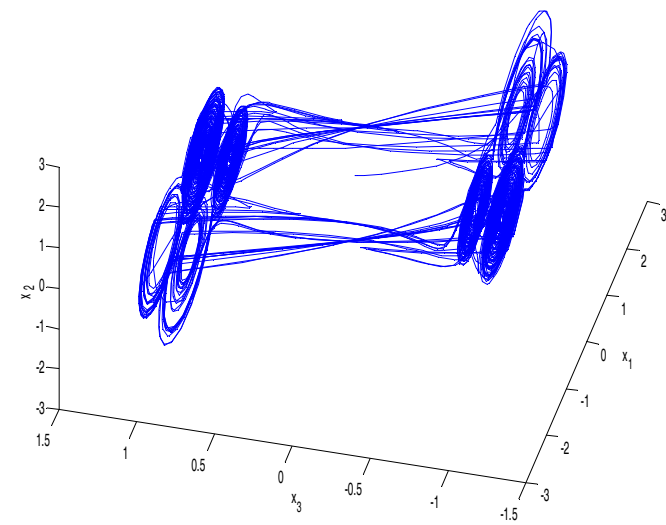

(a)

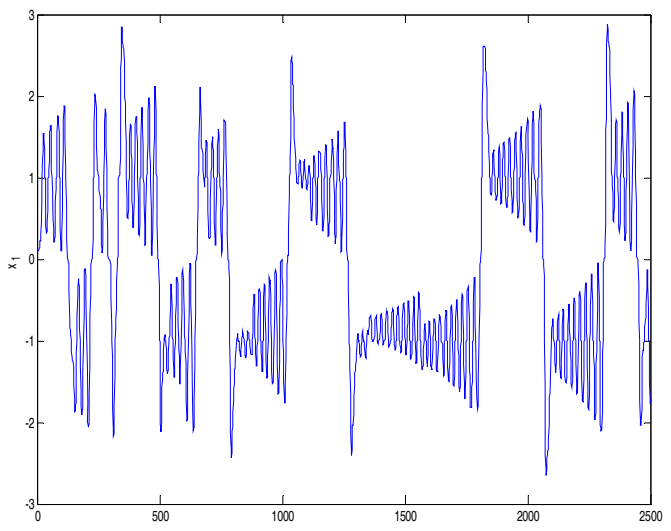

(b)

Figure 9. Cont. 


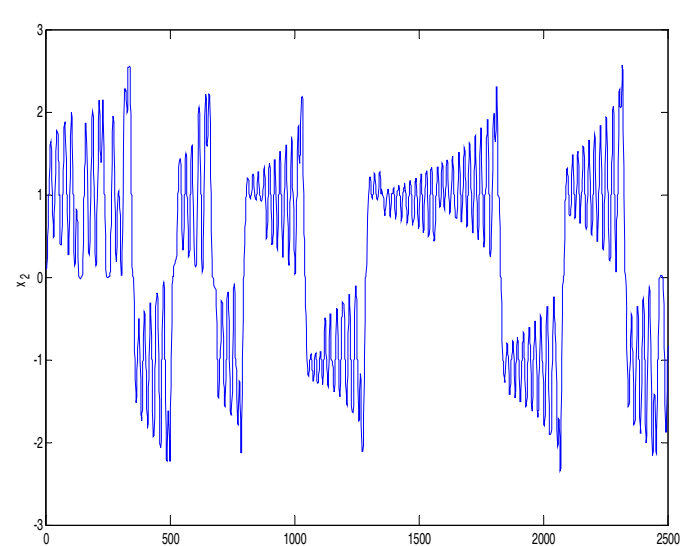

(c)

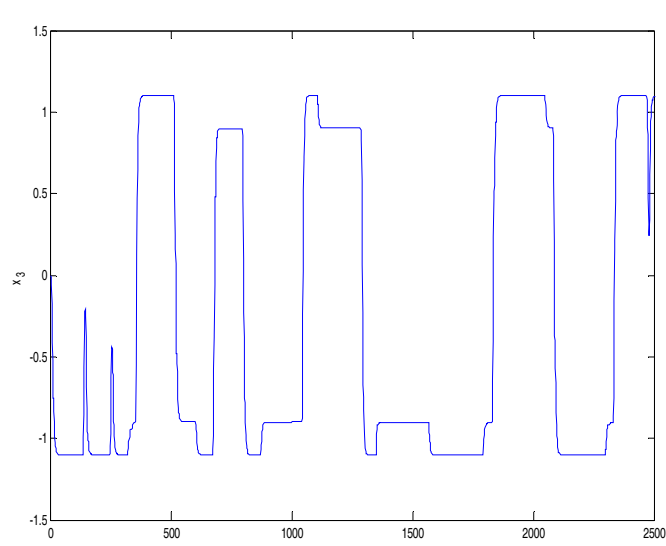

(d)

Figure 9. Numerical results of the $x_{2}-x_{3}$ direction 2-D multi-scroll attractors-II: (a) 4-double scrollin $x_{1}-x_{2}-x_{3}$ plane; $(\mathbf{b})$ variable $x_{1}(\mathrm{t}) ;(\mathbf{c})$ variable $x_{2}(\mathrm{t})$; and $(\mathrm{d})$ variable $x_{3}(\mathrm{t})$.

\subsection{Generating Three Direction 3-D Multi-Scroll Chaotic Attractors}

In this section, we introduce the ways of generating three directions (3-D) multi-scroll chaotic attractors in SC-CNN based system. Hyperbolic tangent function series are added to SC-CNN system along $x_{1}-x_{3}-x_{2}$ directions, $x_{3}-x_{2}-x_{1}$ directions and $x_{3}-x_{1}-x_{2}$ directions, respectively, to generate 3-D multi-scrolls. Equilibrium analysis and their stability are given with phase and time domain responses of each system.

4.3.1. 3-D multi-Scroll Attractors along $x_{1}-x_{3}-x_{2}$ Directions:

$$
\begin{gathered}
\dot{x}_{1}=x_{2}+x_{3}+\tanh \left(n y_{1}\right)+i_{1} \\
\dot{x}_{2}=-x_{1}-x_{2}+s_{22} x_{2}+\tanh \left(n y_{3}\right)+i_{2} \\
\dot{x}_{3}=-x_{3}-\tanh \left(n y_{2}\right)+i_{3} \\
s_{11}=s_{12}=s_{13}=a_{11}=a_{23}=a_{32}=1 ; s_{21}=-1 ; s_{22}=1.05, i_{1}=i_{2}=i_{3}=0.1, n=10 ; \text { and } \\
s_{23}=s_{31}=s_{32}=s_{33}=a_{12}=a_{13}=a_{21}=a_{22}=a_{31}=a_{33}=0 .
\end{gathered}
$$

The equilibrium points of Equation (44) exist in these three subspaces defined as follows:

$$
\begin{gathered}
D_{16}=\left\{\left(x_{1}, x_{2}, x_{3}\right) \mid x_{1}, x_{2}, x_{3} \geq 1\right\}: P_{16}=\left(k_{16}, k_{17}, k_{18}\right), \\
D_{15}=\left\{\left(x_{1}, x_{2}, x_{3}\right)|| x_{1}|,| x_{2}|,| x_{3} \mid \leq 1\right\}: P_{15}=\left(l_{16}, l_{17}, l_{18}\right), \\
D_{17}=\left\{\left(x_{1}, x_{2}, x_{3}\right) \mid x_{1}, x_{2}, x_{3} \leq-1\right\}: P_{17}=\left(m_{16}, m_{17}, m_{18}\right) \\
k_{16}=i_{1}+i_{2}+i_{3}-i_{1} s_{22}-i_{3} s_{22}+1, k_{17}=-i_{3}-i_{1}, k_{18}=i_{3}-1 \\
m_{16}=i_{1}+i_{2}+i_{3}-i_{1} s_{22}-i_{3} s_{22}-1, m_{17}=-i_{3}-i_{1}, m_{18}=i_{3}+1 \\
l_{16}=\frac{-\left(i_{1}+i_{2}+i_{3}-i_{1} s_{22}-i_{3} s_{22}-a_{23} i_{2} n+a_{32} i_{3} n+a_{23} a_{32} i_{1} n^{2}\right.}{\left(a_{11} n+a_{23} n-a_{11} s_{22} n+a_{11} a_{23} a_{32} n^{3}-1\right)}, \\
l_{17}=\frac{\left(a_{11} a_{32} i_{3} n^{2}-a_{1} i_{23} n+i_{1}+i_{3}\right)}{\left(a_{11} n+a_{23} n-a_{11} 1_{22} 2+a_{11} a_{23} 3_{32} n^{3}-1\right)}, \\
l_{18}=\frac{-\left(i_{3}+a_{23} i_{1} n-a_{11} i_{3} n+a_{1} i_{3} s_{22} n+a_{11} a_{23} n^{2}\right)}{\left(a_{11} n+a_{23} n-a_{11} s_{22} n+a_{11} a_{23} a_{32} n^{3}-1\right)}
\end{gathered}
$$

The equilibrium points $P_{15}, P_{16}$, and $P_{17}$ can be found from the Jacobian matrices.

$$
J_{16}=J_{17}=\left[\begin{array}{ccc}
0 & 1 & 1 \\
-1 & \left(s_{22}-1\right) & 0 \\
0 & 0 & -1
\end{array}\right], J_{15}=\left[\begin{array}{ccc}
a_{11} n & 1 & 1 \\
-1 & \left(s_{22}-1\right) & a_{32} n \\
0 & -a_{23} n & 1
\end{array}\right]
$$


The corresponding characteristic equation is:

$$
\begin{gathered}
P_{16}(\lambda)=P_{17}(\lambda)=\lambda^{3}+\left(2-s_{22}\right) \lambda^{2}+\left(2-s_{22}\right) \lambda+1 \\
P_{15}(\lambda)=\lambda^{3}-\left(s_{22}+a_{11} n-2\right) \lambda^{2}-\left(a_{11} n s_{22}-2 a_{11} n-s_{22}+a_{23} a_{32} n^{2}+2\right) \lambda-a_{23} n \\
-a_{11} n+a_{11} s_{22} n-a_{11} a_{23} a_{32} n^{3}+1
\end{gathered}
$$

The system can produce chaotic behaviors for most of the initial conditions and those are taken as $(0.1,0.1,0.1)$ in this paper. The eigenvalues for equilibrium point $(-0.01,0.0111,-0.0111) \in D_{15}$ are calculated from the matrix $J_{15}$ as $\lambda_{1}=9.9952$ and $\lambda_{2,3}=-0.4726 \pm 10.0337 i$, and the eigenvalues for equilibrium points $(1.09,-0.2,-0.9) \in D_{16}$ and $(-0.91,-0.2,1.1) \in D_{17}$ are calculated from the matrix $J_{16,17}$ as $\lambda_{1}=-1$ and $\lambda_{2,3}=0.0250 \pm 0.9997 i$. System (44) has one positive root and one pair of complex conjugate roots with negative real parts for $D_{15}$ subspace, and SC-CNN System (44) becomes unstable and equilibria $P_{15}$ is a saddle point index 1 . On the other hand, System (44) has one negative root and one pair of complex conjugate roots with positive real parts for $D_{16,17}$ subspaces. Thus, SC-CNN System (44) becomes unstable and equilibrium points $P_{16,17}$ are saddle point index 2 .

$$
\begin{aligned}
& \left.\begin{array}{rl}
\lambda_{1}+\lambda_{2}+\lambda_{3} & =\left(s_{22}-2\right)<0 \\
\lambda_{1} \lambda_{2} \lambda_{3} & =-1<0
\end{array}\right\} \text { for } D_{16}, D_{17} \\
& \left.\begin{array}{l}
\lambda_{1}+\lambda_{2}+\lambda_{3}=\left(s_{22}+a_{11} n-2\right)>0 \\
=\left[\left(a_{11}+a_{23}-a_{11} s_{22}\right) n+a_{11} a_{23} a_{32} n^{3}-1\right]>0
\end{array}\right\} \text { for } D_{15}
\end{aligned}
$$

Figure 10a shows $x_{1}-x_{2}-x_{3}$ plane projection of 2-double-scroll attractor.

Remark 6. System (44) can generate 3-D multi-scroll chaotic attractors by:

- adding tanh functions in $x_{1}$ direction via $y_{1}$ nonlinear function, and in $x_{2}$ direction via $y_{3}$ nonlinear function, and in $x_{3}$ direction via $y_{2}$ nonlinear function; and

- choosing parameters $s_{22}, n, a_{11}, a_{23}, a_{32}$ to satisfy condition given in Equation (49).

Figure 10b presents time domain responses of $x_{1}(\mathrm{t})$, Figure 10c,d presents 4-double-scroll attractor and time response of $x_{3}(t)$ for:

$$
\begin{gathered}
\dot{x}_{1}=x_{2}+x_{3}+\tanh \left(n y_{1}\right)+i_{1 a}+\tanh \left(n y_{1}\right)-i_{1 a}+ \\
\tanh \left(n y_{1}\right)+i_{1 b}+\tanh \left(n y_{1}\right)-i_{1 b} \\
\dot{x}_{2}=-x_{1}-x_{2}+s_{22} x_{2}+\tanh \left(n y_{3}\right)+i_{2 a}+\tanh \left(n y_{3}\right)-i_{2 a}+ \\
\tanh \left(n y_{3}\right)+i_{2 b}+\tanh \left(n y_{3}\right)-i_{2 b} \\
\dot{x}_{3}=-x_{3}-\tanh \left(n y_{2}\right)+i_{3 a}-\tanh \left(n y_{2}\right)-i_{3 a}- \\
\tanh \left(n y_{2}\right)+i_{3 b}-\tanh \left(n y_{2}\right)-i_{3 b}
\end{gathered}
$$

where $i_{1 a}=i_{2 a}=i_{3 a}=0.1, i_{1 b}=0.3$, and $i_{2 b}=i_{3 b}=0.2$. 


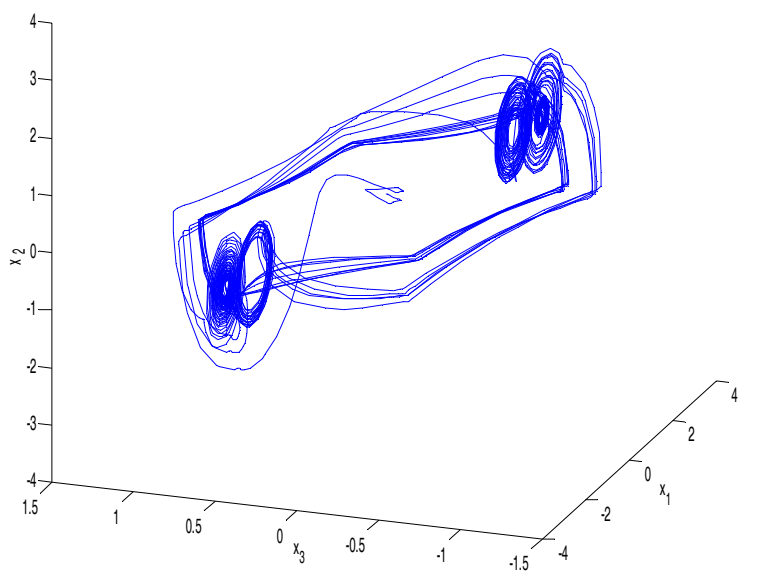

(a)

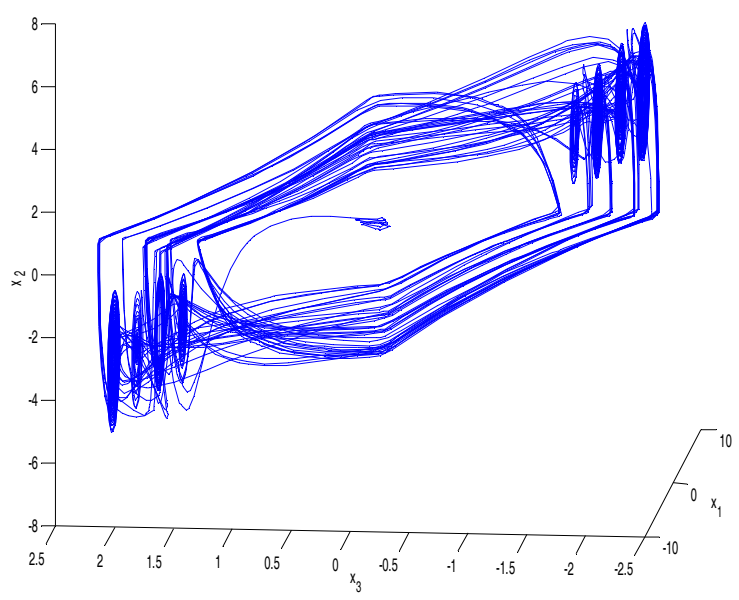

(c)

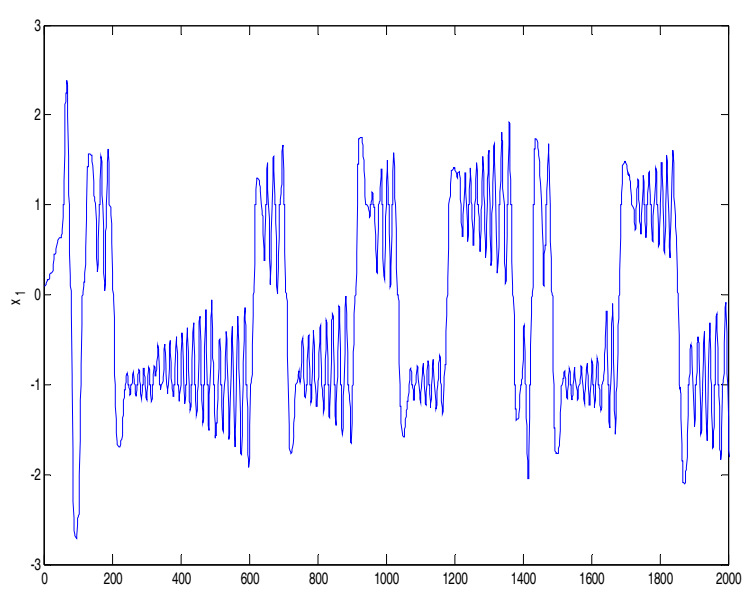

(b)

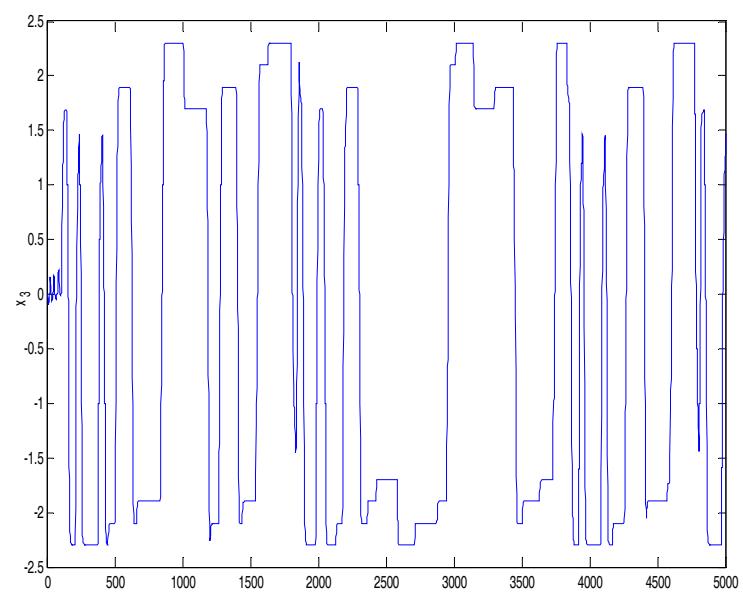

(d)

Figure 10. Numerical results of the 3-D multi-scroll attractors-I: (a) 2-double scrollin $x_{1}-x_{2}-x_{3}$ plane; (b) variable $x_{1}(\mathrm{t})$; (c) 4-double scrollin $x_{1}-x_{2}-x_{3}$ plane; and (d) variable $x_{3}(\mathrm{t})$.

\subsubsection{3-D Multi-Scroll Attractors along $x_{3}-x_{2}-x_{1}$ Directions:}

$$
\begin{gathered}
\dot{x}_{1}=x_{2}+x_{3}+\tanh \left(n y_{3}\right)+i_{1} \\
\dot{x}_{2}=-x_{1}-x_{2}+s_{22} x_{2}+\tanh \left(n y_{2}\right)+i_{2} \\
\dot{x}_{3}=-x_{3}-\tanh \left(n y_{1}\right)+i_{3} \\
s_{11}=s_{12}=s_{13}=a_{13}=a_{22}=a_{31}=1 ; s_{21}=-1 ; s_{22}=1.1, i_{1}=i_{2}=i_{3}=0.1, n=10 ; \text { and } \\
s_{23}=s_{31}=s_{32}=s_{33}=a_{11}=a_{12}=a_{21}=a_{23}=a_{32}=a_{33}=0 .
\end{gathered}
$$

The equilibrium points of Equation (48) exist in these three subspaces, defined as follows:

$$
\begin{gathered}
D_{19}=\left\{\left(x_{1}, x_{2}, x_{3}\right) \mid x_{1}, x_{2}, x_{3} \geq 1\right\}: P_{19}=\left(k_{19}, k_{20}, k_{21}\right), \\
D_{18}=\left\{\left(x_{1}, x_{2}, x_{3}\right)|| x_{1}|,| x_{2}|,| x_{3} \mid \leq 1\right\}: P_{18}=\left(l_{19}, l_{20}, l_{21}\right), \\
D_{20}=\left\{\left(x_{1}, x_{2}, x_{3}\right) \mid x_{1}, x_{2}, x_{3} \leq-1\right\}: P_{20}=\left(m_{19}, m_{20}, m_{21}\right)
\end{gathered}
$$




$$
\begin{gathered}
k_{19}=i_{1}+i_{2}+i_{3}-i_{1} s_{22}-i_{3} s_{22}+1, k_{20}=-i_{3}-i_{1}, k_{21}=i_{3}-1 \\
m_{19}=i_{1}+i_{2}+i_{3}-i_{1} s_{22}-i_{3} s_{22}-1, m_{20}=-i_{3}-i_{1}, m_{21}=i_{3}+1 \\
l_{19}=\frac{-\left(i_{1} s_{22}-i_{2}-i_{3}-i_{1}+i_{3} s_{22}+a_{22} i_{1} n+a_{22} i_{3} n-a_{31} i_{3} n+a_{31} i_{3} s_{22} n+a_{22} a_{31} i_{3} n^{2}\right)}{\left(a_{13} n s_{22}-a_{13} n+a_{13} a_{22} n^{2}-a_{13} a_{31} n^{2}+a_{13} a_{22} a_{31} n^{3}+a_{13} a_{31} s_{22} n^{2}+1\right)}, \\
l_{20}=\frac{-\left(i_{1}+i_{3}+a_{13} i_{2} n+a_{31} i_{3} n+a_{13} a_{31} i_{2} n^{2}\right)}{\left(a_{13} n s_{22}-a_{13} n+a_{13} a_{22} n^{2}-a_{13} a_{31} n^{2}+a_{13} a_{22} a_{31} n^{3}+a_{13} a_{31} s_{22} n^{2}+1\right)}, \\
l_{21}=\frac{\left(i_{3}+a_{13} i_{1} n+a_{13} i_{2} n-a_{13} i_{1} s_{22} n-a_{13} a_{22} i_{1} n^{2}\right)}{\left(a_{13} n s_{22}-a_{13} n+a_{13} a_{22} n^{2}-a_{13} a_{31} n^{2}+a_{13} a_{22} a_{31} n^{3}+a_{13} a_{31} s_{22} n^{2}+1\right)}
\end{gathered}
$$

The equilibrium points $P_{18}, P_{19}, P_{20}$ can be found through the Jacobian matrices.

$$
J_{19}=J_{20}=\left[\begin{array}{ccc}
0 & 1 & 1 \\
-1 & \left(s_{22}-1\right) & 0 \\
0 & 0 & -1
\end{array}\right] ; J_{18}=\left[\begin{array}{ccc}
0 & 1 & a_{31} n+1 \\
-1 & \left(s_{22}+a_{2} n-1\right) & 0 \\
a_{13} n & 0 & -1
\end{array}\right]
$$

The corresponding characteristic equation is:

$$
\left.\begin{array}{c}
\left.\begin{array}{c}
P_{19}(\lambda)=P_{20}(\lambda)=\lambda^{3}+\left(2-s_{22}\right) \lambda^{2}+\left(2-s_{22}\right) \lambda+1 \\
P_{18}(\lambda)=\lambda^{3}-\left(s_{22}+a_{22} n-2\right) \lambda^{2}-\left(s_{22}+a_{13} n+a_{22} n+a_{11} a_{31} n^{2}-2\right) \lambda \\
-a_{13} n+a_{13} s_{22} n+a_{13} a_{22} n^{2}-a_{13} a_{31} n^{2}+a_{13} a_{22} a_{31} n^{3}+a_{13} a_{31} s_{22} n^{2}+1 \\
\lambda_{1}+\lambda_{2}+\lambda_{3}=\left(s_{22}-2\right)<0 \\
\lambda_{1} \lambda_{2} \lambda_{3}=-1<0
\end{array}\right\} \text { for } D_{19}, D_{20} \\
\lambda_{1}+\lambda_{2}+\lambda_{3}=\left(s_{22}+a_{22} n-2\right)>0 \\
\lambda_{1} \lambda_{2} \lambda_{3}=\left[\begin{array}{c}
\left(a_{13}-a_{13} s_{22}\right) n+\left(a_{13} a_{31}-a_{13} a_{22}-a_{13} s_{22} a_{31}\right) n^{2} \\
-\left(a_{13} a_{22} a_{31}\right) n^{3}-1
\end{array}\right]>0
\end{array}\right\} \text { for } D_{18}
$$

Initial conditions are taken as $(0.1,0.1,0.1)$ and the equilibrium points and eigenvalues of the Jacobian matrices are calculated for the parameter values as $s_{11}=s_{12}=s_{13}=a_{13}=a_{22}=a_{31}=1 ; s_{21}=-1$; $s_{22}=1.1, i_{1}=i_{2}=i_{3}=0.1$, and $n=10$. The eigenvalues for equilibrium point $(-0.0108,-0.0110,0.0081)$ $\in D_{18}$ are calculated from the matrix $J_{18}$ as $\lambda_{1}=-10.9774$ and $\lambda_{2,3}=10.0387 \pm 0.7231 i$; the eigenvalues for equilibrium points $(1.09,-0.2,-0.9) \in D_{19}$ are calculated from the matrix $J_{19}$ as $\lambda_{1}=-1$ and $\lambda_{2,3}=$ $0.0250 \pm 0.9997 i$; and $(-0.92,-0.2,1.1) \in D_{20}$ are calculated from the matrix $J_{20}$ as $\lambda_{1}=-1$ and $\lambda_{2,3}$ $=0.0500 \pm 0.9987 \mathrm{i}$. System (51) has one negative root and one pair of complex conjugate roots with positive real parts for all subspaces.

Remark 7. System (51) can generate 3-D multi-scroll chaotic attractors by:

- $\quad$ adding tanh functions in $x_{1}$ direction via $y_{3}$ nonlinear function, and in $x_{2}$ direction via $y_{2}$ nonlinear function, and in $x_{3}$ direction via $y_{1}$ nonlinear function; and

- $\quad$ choosing parameters $s_{22}, n, a_{13}, a_{22}, a_{31}$ according to satisfy condition Equation (56)

System (51) is unstable and all equilibrium points $P$ are saddle points of index 2 . Figure 11 a shows $x_{1}-x_{2}-x_{3}$ plane projection of 2 -double-scroll attractor. Figure $11 \mathrm{~b}$ presents time domain responses of $x_{1}(\mathrm{t})$, Figure $11 \mathrm{c}, \mathrm{d}$ presents 4 -double-scroll attractor and time response of $x_{3}(\mathrm{t})$ for:

$$
\begin{gathered}
\dot{x}_{1}=x_{2}+x_{3}+\tanh \left(n y_{3}\right)+i_{1 a}+\tanh \left(n y_{3}\right)-i_{1 a}+ \\
\tanh \left(n y_{3}\right)+i_{1 b}+\tanh \left(n y_{3}\right)-i_{1 b} \\
\dot{x}_{2}=-x_{1}-x_{2}+s_{22} x_{2}+\tanh \left(n y_{2}\right)+i_{2 a}+\tanh \left(n y_{2}\right)-i_{2 a}+ \\
\tanh \left(n y_{2}\right)+i_{2 b}+\tanh \left(n y_{2}\right)-i_{2 b} \\
\dot{x}_{3}=-x_{3}-\tanh \left(n y_{1}\right)+i_{3 a}-\tanh \left(n y_{1}\right)-i_{3 a}-\tanh \left(n y_{1}\right)+i_{3 b}-\tanh \left(n y_{1}\right)-i_{3 b}
\end{gathered}
$$

where $i_{1 a}=i_{2 a}=i_{3 a}=0.2$, and $i_{1 b}=i_{2 b}=i_{3 b}=0.3$. 


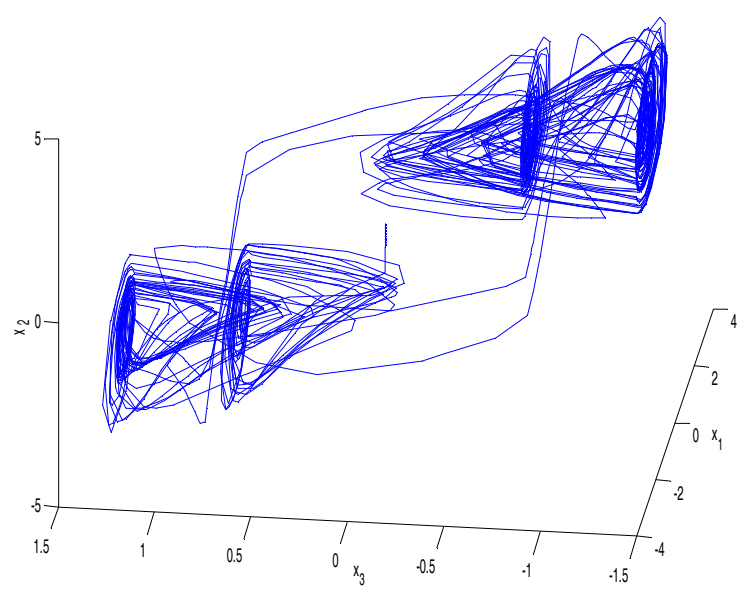

(a)

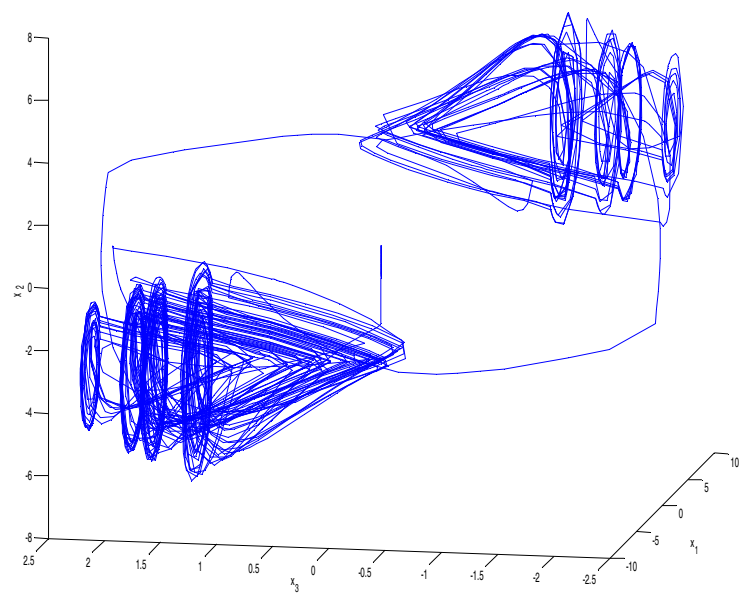

(c)

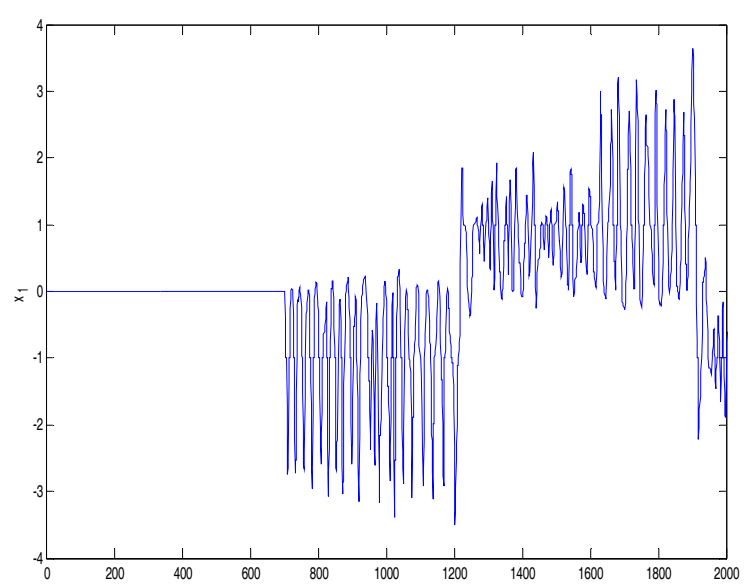

(b)

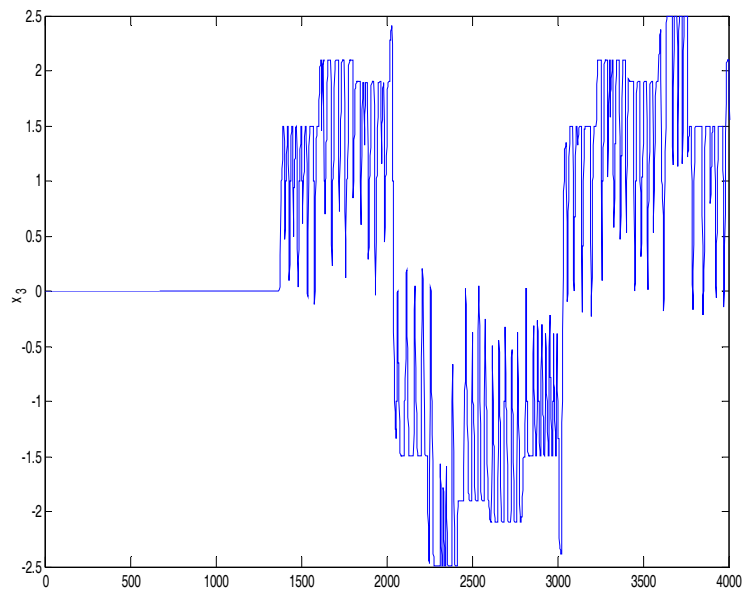

(d)

Figure 11. Numerical results of the 3-D multi-scroll attractors-II: (a) 2-double scrollin $x_{1}-x_{2}-x_{3}$ plane; (b) variable $x_{1}(\mathrm{t})$; (c) 4-double scrollin $x_{1}-x_{2}-x_{3}$ plane; and (d) variable $x_{3}(\mathrm{t})$.

4.3.3. 3-D Multi-Scroll Attractors along $x_{3}-x_{1}-x_{2}$ Directions:

$$
\begin{gathered}
\dot{x}_{1}=x_{2}+x_{3}+\tanh \left(n y_{3}\right)+i_{1} \\
\dot{x}_{2}=-x_{1}-x_{2}+s_{22} x_{2}+\tanh \left(n y_{1}\right)+i_{2} \\
\dot{x}_{3}=-x_{3}-\tanh \left(n y_{2}\right)+i_{3} \\
s_{11}=s_{12}=s_{13}=a_{13}=a_{21}=a_{32}=1 ; s_{21}=-1 ; s_{22}=1.1, i_{1}=i_{2}=i_{3}=0.1, n=10 ; \text { and } \\
s_{23}=s_{31}=s_{32}=s_{33}=a_{11}=a_{12}=a_{22}=a_{23}=a_{31}=a_{33}=0 .
\end{gathered}
$$

The equilibrium points of Equation (53) exist in these three subspaces, defined as follows:

$$
\begin{gathered}
D_{22}=\left\{\left(x_{1}, x_{2}, x_{3}\right) \mid x_{1}, x_{2}, x_{3} \geq 1\right\}: P_{22}=\left(k_{22}, k_{23}, k_{24}\right), \\
D_{21}=\left\{\left(x_{1}, x_{2}, x_{3}\right)|| x_{1}|,| x_{2}|,| x_{3} \mid \leq 1\right\}: P_{21}=\left(l_{22}, l_{23}, l_{24}\right), \\
D_{23}=\left\{\left(x_{1}, x_{2}, x_{3}\right) \mid x_{1}, x_{2}, x_{3} \leq-1\right\}: P_{23}=\left(m_{22}, m_{23}, m_{24}\right),
\end{gathered}
$$




$$
\begin{gathered}
k_{22}=i_{1}+i_{2}+i_{3}-i_{1} s_{22}-i_{3} s_{22}+1, \quad k_{23}=-i_{3}-i_{1}, \quad k_{24}=i_{3}-1 \\
m_{22}=i_{1}+i_{2}+i_{3}-i_{1} s_{22}-i_{3} s_{22}-1, m_{23}=-i_{3}-i_{1}, m_{24}=i_{3}+1 \\
l_{22}=\frac{-\left(i_{1} s_{22}-i_{2}-i_{3}-i_{1}+i_{3} s_{22}+a_{22} i_{2} n-a_{32} i_{3} n+a_{32} i_{3} s_{22} n+a_{21} a_{32} i_{2} n^{2}\right)}{\left(a_{13} n-1\right)\left(a_{2} a_{32} n^{2}+a_{21} n-1\right)}, \\
l_{23}=\frac{\left(i_{1}+i_{3}+a_{32} i_{3} n\right)^{1}}{\left(a_{21} a_{32} n^{2}+a_{21} n-1\right)} \\
l_{21}=\frac{-\left(i_{3}+a_{1} i_{1} n\right)}{\left(a_{21} a_{32} n^{2}+a_{21} n-1\right)}
\end{gathered}
$$

The equilibrium points $P_{21}, P_{22}$, and $P_{23}$ can be found from the Jacobian matrices.

$$
J_{22}=J_{23}=\left[\begin{array}{ccc}
0 & 1 & 1 \\
-1 & \left(s_{22}-1\right) & 0 \\
0 & 0 & -1
\end{array}\right] J_{21}=\left[\begin{array}{ccc}
0 & 1 & \left(a_{32} n+1\right) \\
\left(a_{13} n-1\right) & \left(s_{22}-1\right) & 0 \\
0 & -a_{21} n & -1
\end{array}\right]
$$

The corresponding characteristic equation is:

$$
\begin{aligned}
& P_{22}(\lambda)=P_{23}(\lambda)=\lambda^{3}+\left(2-s_{22}\right) \lambda^{2}+\left(2-s_{22}\right) \lambda+1 \\
& P_{21}(\lambda)=\lambda^{3}-\left(s_{22}-2\right) \lambda^{2}-\left(s_{22}+a_{13} n-2\right) \lambda-a_{13} n-a_{21} n+a_{13} a_{21} n^{2}-a_{21} a_{32} n^{2}+a_{13} a_{21} a_{32} n^{3}+1 \\
& \left.\begin{array}{rl}
\lambda_{1}+\lambda_{2}+\lambda_{3} & =\left(s_{22}-2\right)<0 \\
\lambda_{1} \lambda_{2} \lambda_{3} & =-1<0
\end{array}\right\} \text { for } D_{22}, D_{23} \\
& \left.\begin{array}{c}
\lambda_{1}+\lambda_{2}+\lambda_{3}=\left(s_{22}-2\right)<0 \\
\lambda_{1} \lambda_{2} \lambda_{3}=\left(a_{13} n-1\right)\left(a_{21} a_{32} n^{2}+a_{21} n-1\right)<0
\end{array}\right\} \text { for } D_{21}
\end{aligned}
$$

The system can produce chaotic behaviors for most of the initial conditions and those are taken as $(0.1,0.1,0.1)$ in this paper. The equilibrium points and eigenvalues of the Jacobian matrices are calculated for the parameter values $s_{11}=s_{12}=s_{13}=a_{13}=a_{21}=a_{32}=1 ; s_{21}=-1 ; s_{22}=1.1 ; i_{1}=i_{2}=$ $i_{3}=0.1$; and $n=10$. The eigenvalues for equilibrium point $(-0.06,-0.05,0.05) \in D_{21}$ are calculated from the matrix $J_{21}$ as $\lambda_{1}=-1.2469$ and $\lambda_{2,3}=0.7234 \pm 1.0396 i$, and the eigenvalues for equilibrium points $(-1.32,-1.1,1.1) \in D_{22}$ and $(1.08,0.9,-0.9) \in D_{23}$ are calculated from the matrix $J_{1,2}$ as $\lambda_{1}=$ -1 and $\lambda_{2,3}=0.6 \pm 0.8 i$. System (58) has one negative root and one pair of complex conjugate roots with positive real parts for all subspaces. Then, System (58) is unstable and all equilibrium points $P$ are saddle points of index 2 .

Remark 8. The two strategies that can generate 3-D multi-scroll chaotic attractors in System (58) are summarized as follows:

- adding tanh functions in $x_{1}$ direction via $y_{3}$ nonlinear function, and in $x_{2}$ direction via $y_{1}$ nonlinear function, and in $x_{3}$ direction via $y_{2}$ nonlinear function; and

- $\quad$ arameterss $22, n, a_{13}, a_{21}, a_{32}$ satisfy condition given in Equation (63).

Figure 12a shows $x_{1}-x_{2}-x_{3}$ plane projection of 4-double-scroll attractor. Figure $12 \mathrm{~b}$ presents time domain responses of $x_{1}(\mathrm{t})$, Figure 12c,d presents 8-double-scroll attractor and time response of $x_{3}(\mathrm{t})$ for:

$$
\begin{gathered}
\dot{x}_{1}=x_{2}+x_{3}+\tanh \left(n y_{3}\right)+i_{1 a}+\tanh \left(n y_{3}\right)-i_{1 a}+ \\
\tanh \left(n y_{3}\right)+i_{1 b}+\tanh \left(n y_{3}\right)-i_{1 b} \\
\dot{x}_{2}=-x_{1}-x_{2}+s_{22} x_{2}+\tanh \left(n y_{1}\right)+i_{2 a}+\tanh \left(n y_{1}\right)-i_{2 a}+ \\
\tanh \left(n y_{1}\right)+i_{2 b}+\tanh \left(n y_{1}\right)-i_{2 b} \\
\dot{x}_{3}=-x_{3}-\tanh \left(n y_{2}\right)+i_{3 a}-\tanh \left(n y_{2}\right)-i_{3 a}- \\
\tanh \left(n y_{2}\right)+i_{3 b}-\tanh \left(n y_{2}\right)-i_{3 b}
\end{gathered}
$$

where $i_{1 a}=i_{2 a}=i_{3 a}=0.1$, and $i_{1 b}=i_{2 b}=i_{3 b}=0.2$. 


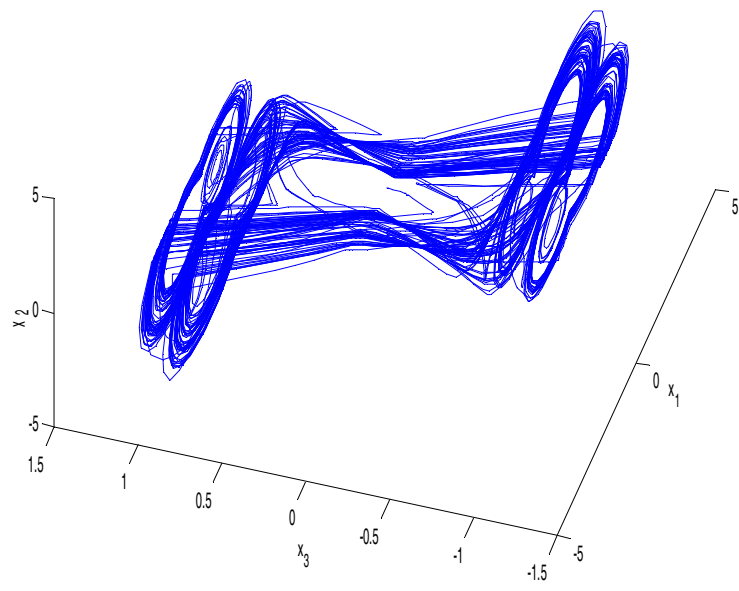

(a)

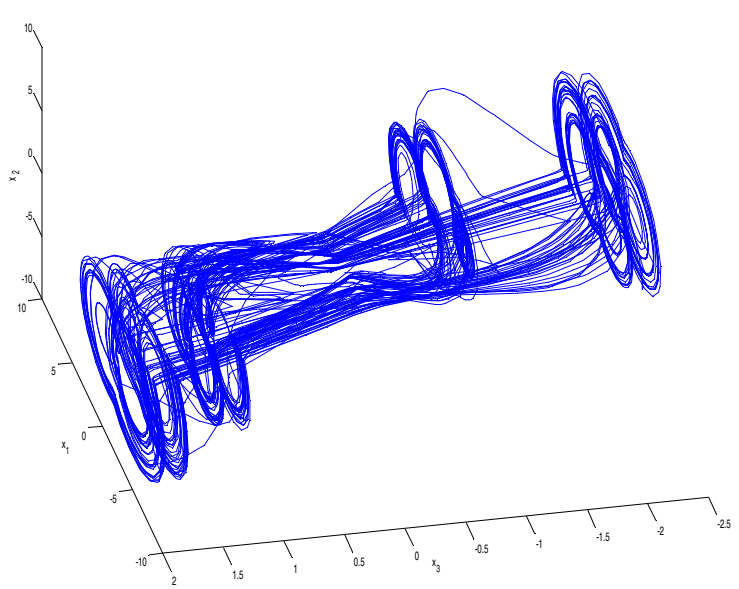

(c)

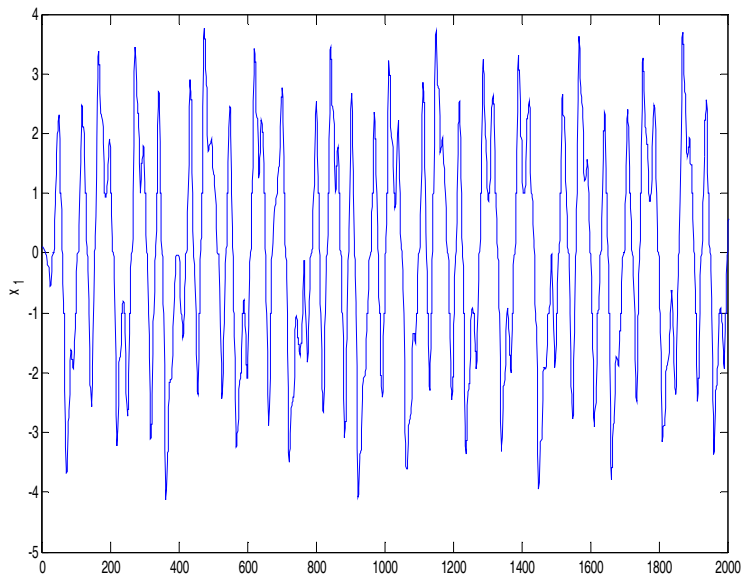

(b)

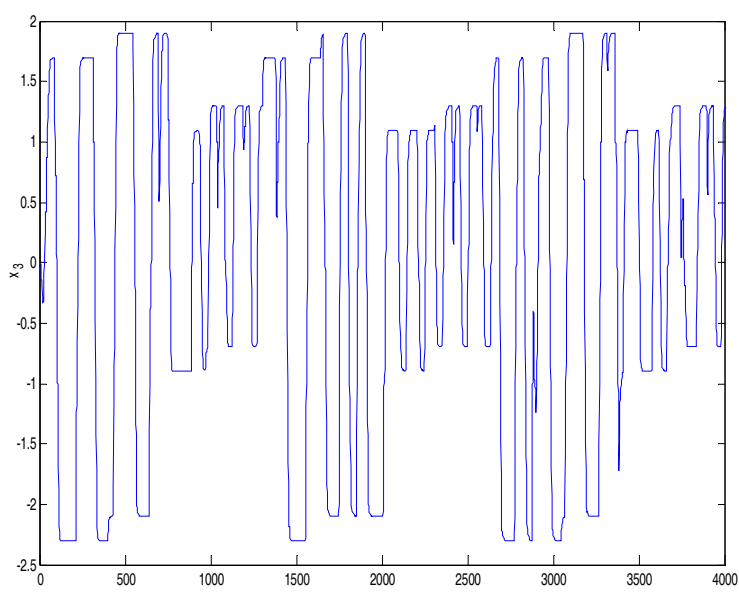

(d)

Figure 12. Numerical results of the 3-D multi-scroll attractors-III: (a) 2-double scrollin $x_{1}-x_{2}-x_{3}$ plane; (b) variable $x_{1}(\mathrm{t})$; (c) 8 -double scrollin $x_{1}-x_{2}-x_{3}$ plane; and (d) variable $x_{3}(\mathrm{t})$.

\section{Conclusions}

In this paper, a new SC-CNN based chaotic system with multiple hyperbolic tangent functions is proposed. This paper presents a mathematical approach for generating multi-scroll chaotic attractors, such as (one-directional) 1-D, (two-directional) 2-D scroll, and (three-directional) 3-D scroll attractors, from a given 3D linear autonomous SC-CNN system with a hyperbolic function series as the controller. The mechanism for generating multi-scroll chaotic attractors is theoretically analyzed and numerically simulated. Some dynamical behaviors of this system are investigated, such as their equilibria, stability, Lyapunov exponents and bifurcation diagrams. A Poincaré map, particularly is constructed for verifying the chaotic behaviors of the double-scroll attractor.

The system under consideration displays complex nonlinear phenomena as period doubling bifurcation and multi-scroll generation for different sets of system parameters. The model considered in this work represents an interesting tool for students and researchers to learn better about nonlinear dynamics and chaos. Furthermore, this system can be widely evaluated in data encryption and signal communication. Finally, it can be predicted that various related bifurcation phenomena in the generated multi-scroll chaotic systems need to be further investigated in the near future.

Author Contributions: E.G. and K.A. performed theoretical and numerical analysis.

Funding: This work is supported by Research Fund of Erciyes University (Project Code: FDK-2016-6757). 
Conflicts of Interest: The authors declare no conflict of interest.

\section{References}

1. Landaw, J.; Garfinkel, A.; Weiss, J.N.; Qu, Z. Memory-induced chaos in cardiac excitation. Phys. Rev. Lett. 2017, 118, 138101. [CrossRef] [PubMed]

2. Chen, G.; Ueta, T. Chaos in Circuits and Systems, World Scientific Series on Nonlinear Science; Series B (Book 11); World Scientific Publishing: Singapore, 2002.

3. Chua, L.O.; Yang, L. Cellular neural networks: Theory. IEEE Trans. Circuits Syst. 1988, 35, 732-745. [CrossRef]

4. Chua, L.O. CNN: A Paradigm for complexity. In World Scientific Series on Nonlinear Science; Series A; World Scientific Publishing: Singapore, 1998; Volume 31.

5. Suykens, J.A.K.; Vandewalle, J. Quasilinear approach to nonlinear systems and the design of n-double scroll ( $\mathrm{n}=1,2,3,4, \ldots)$. IEE Proc. G 1991, 138, 595-603.

6. Suykens, J.A.K.; Vandewalle, J. Generation of $\mathrm{n}$-double scrolls ( $\mathrm{n}=1,2,3,4, \ldots)$. IEEE Trans. Circuits Syst. I 1993, 40, 861-867. [CrossRef]

7. Suykens, J.A.K.; Vandewalle, J. Between $n$-double sinks and $n$-double scrolls ( $\mathrm{n}=1,2,3,4, \ldots)$. In Proceedings of the International Symposium on Nonlinear Theory and its Application (NOLTA'93), Honolulu, HI, USA, 5-10 December 1993; pp. 829-834.

8. Aziz-Alaoui, M.A. Differential equations with multispiral attractors. Int. J. Bifurc. Chaos 1999, 9, $1009-1039$. [CrossRef]

9. Aziz-Alaoui, M.A. Multispiral chaos. In Proceedings of the 2nd International Conference Control Oscillations and Chaos, St. Petersberg, Russsia, 5-7 July 2000; pp. 88-91.

10. Yalçı, M.E.; Suykens, J.A.K.; Vandewalle, J. Hyperchaotic n-scroll attractors. In Proceedings of the IEEE Workshop on Nonlinear Dynamics of Electronic Systems (NDES'00), Catania, Italy, 18-20 May 2000; pp. 25-28.

11. Yalçın, M.E.; Özoğuz, S.; Suykens, J.A.K.; Vandewalle, J. n-scroll chaos generators: A simple circuit model. Electron. Lett. 2001, 37, 147-148. [CrossRef]

12. Lü, J.; Chen, G.; Yu, X.; Leung, H. Generating multi-scroll chaotic attractors via switching control. In Proceedings of the 5th Asian Control Conference, Melbourne, Australia, 20-23 July 2004; pp. 1763-1771.

13. Lü, J.; Chen, G.; Yu, X.; Leung, H. Design and analysis of multi-scroll chaotic attractors from saturated function series. IEEE Trans. Circuits Syst. I 2004, 51, 2476-2490. [CrossRef]

14. Lü, J.; Chen, G. Generating multi scroll chaotic attractors: Theories, methods and applications. Int. J. Bifurc. Chaos 2006, 16, 775-858. [CrossRef]

15. Tang, K.S.; Zhong, G.Q.; Chen, G.; Man, K.F. Generation of n-scroll attractors via sine function. IEEE Trans. Circuits Syst. I 2001, 48, 1369-1372. [CrossRef]

16. Özoğuz, S.; Elwakil, A.S.; Salama, K.N. n-Scroll chaos generator using nonlinear transconductor. Electron. Lett. 2002, 38, 685-686. [CrossRef]

17. Salama, K.N.; Özoğuz, S.; Elwakil, A.S. Generation of n-scroll chaos using nonlinear transconductors. In Proceedings of the IEEE International Symposium Circuits and Systems (ISCAS'03), Bangkok, Thailand, 25-28 May 2003; pp. 176-179.

18. Cafagna, D.; Grassi, G. Hyperchaotic coupled Chua circuits: An approach for generating new $\mathrm{n} \times \mathrm{m}$ scroll attractors. Int. J. Bifurc. Chaos 2003, 13, 2537-2550. [CrossRef]

19. Cafagna, D.; Grassi, G. New 3D-scroll attractors in hyperchaotic Chua's circuit forming a ring. Int. J. Bifurc. Chaos 2003, 13, 2889-2903. [CrossRef]

20. Yalçın, M.E.; Suykens, J.A.K.; Vandewalle, J.; Özoğuz, S. Families of scroll grid attractors. Int. J. Bifurc. Chaos 2002, 12, 23-41. [CrossRef]

21. Yu, S.M.; Lü, J.; Leung, H.; Chen, G. Design and circuit implementation of n-scroll chaotic attractor from a general jerk system. IEEE Trans. Circuits Syst. I 2005, 52, 1459-1476.

22. Yu, S.M.; Lü, J.; Leung, H.; Chen, G. N-scroll chaotic attractors from a general jerk circuit. In Proceedings of the IEEE International Symposium Circuits and Systems (ISCAS'05), Kobe, Japan, 23-26 May 2005; pp. 1473-1476.

23. Deng, W. Generating 3-D scroll grid attractors of fractional differential systems via stair function. Int. J. Bifurc. Chaos 2007, 17, 3965-3983. [CrossRef] 
24. Deng, W.; Lü, J. Design of multidirectional multiscroll chaotic attractors based on fractional differential systems via switching control. Chaos Interdiscip. J. Nonlinear Sci. 2006, 16, 43120. [CrossRef] [PubMed]

25. Deng, W.; Lü, J. Generating multi-directional multi-scroll chaotic attractors via a fractional differential hysteresis system. Phys. Lett. A 2007, 369, 438-443. [CrossRef]

26. Chen, L.; Pan, W.; Wu, R.; Wang, K.; He, Y. Generation and circuit implementation of fractional-order multi-scroll attractors. Chaos Solitons Fractals 2016, 85, 22-31. [CrossRef]

27. Chen, L.; Pan, W.; Wu, R.; Machado, J.A.T.; Lopes, A.M. Design and implementation of grid multi-scroll fractional-order chaotic attractors. Chaos Interdiscip. J. Nonlinear Sci. 2016, 26, 84303. [CrossRef] [PubMed]

28. Xu, F.; Yu, P. Chaos control and chaos synchronization for multi-scroll chaotic attractors generated using hyperbolic functions. J. Math. Anal. Appl. 2010, 362, 252-274. [CrossRef]

29. Chen, Z.; Wen, G.; Zhou, H.; Chen, J. Generation of grid multi-scroll chaotic attractors via hyperbolic tangent function series. Opt. Int. J. Light Elect. Opt. 2017, 130, 594-600. [CrossRef]

30. Wang, N.; Bao, B.-C.; Xu, Q.; Chen, M.; Wu, P.Y. Emerging multi-double-scroll attractor from variable-boostable chaotic system excited by multi-level pulse. J. Eng. 2018, 2018, 42-44. [CrossRef]

31. Muñoz-Pacheco, J.M.; Guevara-Flores, D.K.; Félix-Beltrán, O.G.; Tlelo-Cuautle, E.; Barradas-Guevara, J.E.; Volos, C.K. Experimental Verification of Optimized Multiscroll Chaotic Oscillators Based on Irregular Saturated Functions. Complexity 2018, 2018. [CrossRef]

32. Arena, P.; Baglio, S.; Fortuna, L.; Manganaro, G. Generation of n-double scrolls via cellular neural networks. Int. J. Circuit Theory Appl. 1996, 24, 241-252. [CrossRef]

33. Günay, E.; Alçı, M. n-Double Scrolls in SC-CNN Circuit via Diode-Based PWL Function. Int. J. Bifurc. Chaos 2006, 16, 1023-1033. [CrossRef]

34. Günay, E.; Kılıç, R. A New Way of Generating n-Scroll Attractors via Trigonometric Function. Int. J. Bifurc. Chaos 2011, 21, 897-901. [CrossRef]

35. Arena, P.; Baglio, S.; Fortuna, L.; Manganaro, G. Chua's circuit can be generated by CNN cells. IEEE Trans. Circuits Syst. I 1995, 42, 123-125. [CrossRef]

36. Wolf, A.; Swift, J.B.; Swinney, H.L.; Vasano, A. Determining Lyapunov Exponents from A Time Series. Physical D 1985, 16, 285-317. [CrossRef]

(C) 2018 by the authors. Licensee MDPI, Basel, Switzerland. This article is an open access article distributed under the terms and conditions of the Creative Commons Attribution (CC BY) license (http:/ / creativecommons.org/licenses/by/4.0/). 